\title{
Time-frequency component analyser and its application to brain oscillatory activity
}

\author{
Ahmet Kemal Özdemir ${ }^{\mathrm{a}}$, Sirel Karakaş ${ }^{\mathrm{b}, \mathrm{c}, *}$, Emine D. Çakmak ${ }^{\mathrm{b}, \mathrm{c}}$, \\ D. İlhan Tüfekçi ${ }^{\mathrm{a}}$, Orhan Arıkan ${ }^{\mathrm{a}}$ \\ a Bilkent University, Department of Electrical Engineering, 06533 Bilkent, Ankara, Turkey \\ ${ }^{\mathrm{b}}$ Hacettepe University, Specialty Area of Experimental Psychology, 06532 Beytepe, Ankara, Turkey \\ ${ }^{\mathrm{c}}$ The Scientific and Technical Research Council of Turkey, Brain Dynamics Multidisciplinary Research Network, Ankara, Turkey
}

Received 20 February 2004; received in revised form 30 November 2004; accepted 8 December 2004

\begin{abstract}
Currently, event-related potential (ERP) signals are analysed in the time domain (ERP technique) or in the frequency domain (Fourier analysis and variants). In techniques of time-domain and frequency-domain analysis (short-time Fourier transform, wavelet transform) assumptions concerning linearity, stationarity, and templates are made about the brain signals. In the time-frequency component analyser (TFCA), the assumption is that the signal has one or more components with non-overlapping supports in the time-frequency plane. In this study, the TFCA technique was applied to ERPs. TFCA determined and extracted the oscillatory components from the signal and, simultaneously, localized them in the time-frequency plane with high resolution and negligible cross-term contamination. The results obtained by means of TFCA were compared with those obtained by means of other commonly used techniques of ERP analysis, such as bilinear time-frequency distributions and wavelet analysis. It is suggested that TFCA may serve as an appropriate tool for capturing the localized ERP components in the time-frequency domain and for studying the intricate, frequency-based dynamics of the human brain.
\end{abstract}

(C) 2004 Elsevier B.V. All rights reserved.

Keywords: Event-related potentials; Oscillatory brain activity; Brain signal analysis; Time-frequency signal analysis; Component analysis; Biomedical signal processing

\section{Introduction}

The present paper introduces a technique of signal analysis in the time-frequency plane. The technique characterizes the oscillatory components of the complex neuroelectric responses of the brain by identifying and extracting the maximal energies of the oscillatory components and localizing them in the time-frequency plane. It simultaneously displays all significant components in the time-frequency plane and thus presents them in their entirety. The time localization of

\footnotetext{
* Corresponding author. Present address: Hacettepe University, Specialty Area of Experimental Psychology, Beytepe Campus 06532, Ankara, Turkey. Tel.: +90 312297 8335; fax: +90 3122992100 .

E-mail addresses: kozdemir@ieee.org (A.K. Özdemir), skarakas@hacettepe.edu.tr (S. Karakaş).
}

the frequency components is of high resolution and has negligible cross-term contamination. In addition, a comparison of this technique with existing techniques of time-frequency analysis used for electrical signals of the brain is presented.

The brain emits temporally-ordered electrical signals, which can be recorded from the scalp of animals or humans. These electrical fluctuations can be measured as the event-related potentials (ERPs), which are the time-domain responses to external or internal stimuli (Picton et al., 1974; Picton, 1988). The basic technique for ERP waveform analysis is averaging. This technique is used for extracting the components of the evoked ERP from the superimposed, randomly occurring noise and for increasing the signal-to-noise ratio (Dawson, 1954).

Pioneering work on the gamma and alpha oscillations inspired the study of oscillatory activity of the brain (Berger, 
1929; Adrian, 1942). Recently, the analysis of the oscillatory responses of the brain to external or internal stimuli, the event-related oscillations (EROs), has gained much acceptance. Another approach to brain's neuroelectricity has thus become its analysis in the frequency domain. Intensive research shows that the oscillations at various frequencies are valid indices of the brain's information processing operations (for review, see Başar, 1998, 1999; Porjesz et al., 2002; Kamarajan et al., 2004).

The time evolution of the amplitudes, i.e. the ERP waveform alone cannot provide the time localization of the frequency components. Frequency-domain analysis involves the decomposition of ERP into its constituent oscillations (for a review, see Başar, 1980, 1998). Growing amount of research shows that the compound ERP and the ERP components are determined by the superposition of oscillations, called event-related oscillations, in various frequency ranges (Başar, 1980, 1998; Başar et al., 2000; Başar and Ungan, 1973). Karakaş et al. (2000a, 2000b) have demonstrated that, for a series of cognitive paradigms, the amplitudes of the ERP components are determined by a specific combination and phase relationship of oscillatory components, specifically in the delta and theta ranges. The importance of phase relationship of multiple oscillatory components in the production of the average waveform has been demonstrated in the influential study by Makeig et al. (2002). This study showed that the average event-related potential is a combination of phase resetting of ongoing EEG activity with concurrent energy increases. It thus emphasized the importance of oscillatory components and stimulus-induced phase resetting.

One of the widely used methods for demonstrating oscillatory responses of the brain is the transient (evoked) response frequency characteristics method (TRFC). In TRFC, the amplitude-frequency characteristics are computed by the application of one-sided Fourier transform to the transient response (Solodovnikov, 1960; Parvin et al., 1980; Başar, 1980, 1998; Jervis et al., 1983; Brandt and Jansen, 1991; Röschke et al., 1995; Kolev and Yordanova, 1997). Since the amplitude-frequency characteristics are not computed by the successive application of different frequencies, rapid transitions that occur in the brain signal do not present a problem for the TRFC method. The peaks in the amplitude-frequency characteristics (AFC) reveal the resonant frequencies of the system: its excitability and also its response susceptibility (Başar, 1998; Yordanova and Kolev, 1998). The AFC graph thus demonstrates amplitude variations of frequency selectivities. However, it cannot provide the time localization of the components. The technique also assumes that the system studied is linear. Owing to these, the distinctly appearing peaks in TRFC are used in the literature to obtain only a global description of the tuning frequencies of the system (for review, see Başar, 1998, 1999).

Since the oscillatory and non-stationary signal components whose superposition form the ERP waveform are concurrently localized in both the time and frequency domains, time-frequency signal processing is the natural tool for the analysis of non-stationary signals with localized time-frequency supports. Time-frequency distributions (TFDs) are two-dimensional functions that assign the energy content of signals to points in the time-frequency plane (Cohen, 1989). The performance of a TFD is related to its accuracy in describing the signal's energy content in the time-frequency plane, keeping spurious terms negligible. Composite (multi-component) signals, such as biological, acoustic, seismic, speech, radar and sonar signals, whose components have compact time-frequency supports form an important application area for time-frequency signal analysis (Cohen, 1995).

A widely used approximation to time-frequency representation of brain signals is digital filtering (DF). In this method, independent filters are consecutively applied to ERP. Filter limits in DF may be obtained in a response-adaptive way such that the low and high cut-off frequencies of the filters are determined from the frequency range of the resonant selectivities in the corresponding AFC (Cook III and Miller, 1992; Farwell et al., 1993; Başar, 1980). DF thus produces oscillatory components of varying amplitudes within the empirically or theoretically determined filter limits. DF is not well suited to discern the time evolution of an oscillation in a given frequency range in the time-frequency domain.

Another commonly used technique is the wavelet analysis (WA) (Samar et al., 1999). This time-frequency approach is a technique that decomposes the signal into a set of basis functions, called wavelets. If the components of ERP can be represented by using distinct wavelet basis components, then the wavelet decomposition is successful on the desired ERP. When different sizes of wavelets are used, WA may provide a better time-scale localization than DF. Results obtained by WA thus depend on the chosen wavelet prototype. Quadratic B-spline wavelet and orthogonal cubic spline wavelet have proved useful in demonstrating the frequency components in ERP signals (Başar, 1998; Demiralp et al., 1998, 1999, 2001; Başar et al., 1999; Yordonova et al., 2002). Other approaches such as continuous wavelet transform with matching pursuits and wavelet packet models use multiple wavelet prototypes that are selected from a predefined set. The modifications by Rosso et al. (2001) have made it possible to calculate the wavelet entropy and the relative wavelet energy of the different frequency components. Thus, WA provides the time localization of the frequency components. The efficiency of the localization, however, depends on the suitability of the chosen wavelet basis to the complex and highly non-stationary ERPs.

Short-time Fourier transform (STFT) may be a natural choice when analysing the time-frequency characteristics of the ERP signal (Cohen, 1989). However, STFT fails to resolve those ERP components that are closely localized in the time-frequency plane. To increase the resolution of the ERP components in the time-frequency plane, the Wigner distribution can be used (Cohen, 1989). The Wigner distribution 
$W_{x}(t, f)$ of a signal $x(t)$ is defined by the following integral

$W_{x}(t, f)=\int_{-\infty}^{\infty} x\left(t+\frac{t^{\prime}}{2}\right) x^{*}\left(t-\frac{t^{\prime}}{2}\right) \mathrm{e}^{-\mathrm{j} 2 \pi f t^{\prime}} \mathrm{d} t^{\prime}$.

Although the use of Wigner distribution significantly improves the resolution of the individual ERP components, the resultant time-frequency description is heavily cluttered by the cross-terms of the distribution. The cross-terms are oscillatory artefacts in the time-frequency plane. These artefacts may interfere with the auto-components and decrease the interpretability of the Wigner distribution. The cross-terms that occur due to the interaction of different signal components (i.e. auto-components) in a multi-component signal are called outer interference (cross) terms, and the cross-terms that occur due to the interaction of a single-signal component with itself are called inner interference (cross) terms (Fig. 1) (Hlawatsch and Flandrin, 1997). Because of the existence of cross-terms, the Wigner distribution of ERPs cannot provide the desired result.

To overcome cross-term cluttering in the Wigner distribution-based analysis of ERP, a short-time analysis technique has recently been proposed that applies adaptive filters on the Wigner distribution (Jones and Baraniuk, 1995; Tağluk et al., 2002, in press). To emphasize the high frequency features that have low energy, ERP was decomposed into six sub-bands. Using short time, adaptively filtered Wigner distributions, time-frequency analysis was made on each subband. Finally, using a frequency weighting to provide the overall time-frequency representation, the time-frequency distributions corresponding to each of the six sub-band signals were merged. As in all STFT applications, there is a payoff between time and frequency localization. The narrower the chosen time interval, the better the temporal resolution but the poorer the frequency resolution, and vice versa.

Since cross-terms in the Wigner distribution are largeamplitude oscillations, another approach to suppress them is to smooth the Wigner distribution. In a unified framework, the distributions obtained by smoothing the Wigner distribution were studied under the name of Cohen's bilinear class of time-frequency distributions (Cohen, 1989). In this class, the time-frequency distributions of a signal $x(t)$ are given by

$\mathrm{TF}_{x}(t, f)=\int_{-\infty}^{\infty} \int_{-\infty}^{\infty} \kappa(\nu, \tau) A_{x}(\nu, \tau) \mathrm{e}^{-\mathrm{j} 2 \pi(v t+\tau f)} \mathrm{d} \nu \mathrm{d} \tau$,

where $\kappa(\nu, \tau)$ is called the kernel of the transformation (Cohen, 1989, 1995) and $A_{x}(\nu, \tau)$ is the symmetric ambiguity function (AF) which is defined as the two-dimensional inverse Fourier transform (FT) of the Wigner distribution

$$
\begin{aligned}
A_{x}(\nu, \tau) & \triangleq \int_{-\infty}^{\infty} \int_{-\infty}^{\infty} W_{x}(t, f) \mathrm{e}^{\mathrm{j} 2 \pi(v t+\tau f)} \mathrm{d} t \mathrm{~d} f \\
& =\int_{-\infty}^{\infty} x\left(t+\frac{\tau}{2}\right) x^{*}\left(t-\frac{\tau}{2}\right) \mathrm{e}^{\mathrm{j} 2 \pi v t} \mathrm{~d} t .
\end{aligned}
$$

Traditionally, the low-pass smoothing kernel $\kappa(v, \tau)$ is designed to let pass the auto-terms that are centered at the origin of the AF plane, and to suppress the cross-terms that are located away from the origin. The properties of the resulting time-frequency distribution are thus closely related to those of the chosen kernel (for a review of some of this type of time-frequency distributions with fixed kernels, see Page, 1952; Mergenau and Hill, 1961; Choi and Williams, 1989; Cohen, 1989). Usually, these distributions perform well only for a limited class of signals whose auto-terms in the AF plane are located inside the pass-band region of the kernel $\kappa(\nu, \tau)$. For other signals, they offer a trade-off between good cross-term suppression and high auto-term concentration.

To overcome the shortcomings of the TFDs with fixed kernels, TFDs with signal-dependent kernels were proposed (Baraniuk and Jones, 1993; Czerwinski and Jones, 1995). For instance, the well-known optimal radially Gaussian kernel (ORGK) design adaptively chooses the kernel $\kappa(\nu, \tau)$ to cover the auto-terms and to keep cross-terms out of its passband (Baraniuk and Jones, 1993). Signal-dependent TFDs that adapt the pass-band of the kernel to the location of the auto-terms in the AF domain usually offer better cross-term suppression and higher resolution than the TFDs with fixed
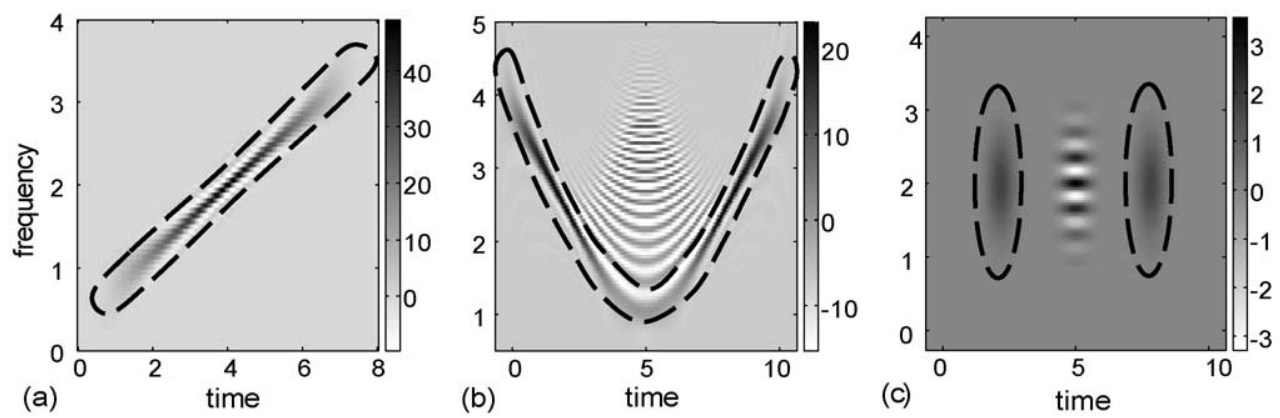

Fig. 1. Wigner distributions of some artificially generated signals. The dashed lines outline the support of the respective auto-components. (a) When the time-frequency support of the signal is convex (a time-frequency support $S$ is called convex if for each pair of its points $A_{i}=\left(t_{i}, f_{i}\right)$ and $B_{j}=\left(t_{j}, f_{j}\right)$ in $S$, the connecting line segment $A_{i} A_{j}$ is also contained in $S$ ), the Wigner distribution has a very high auto-term concentration, and there is negligible crossterm interference. (b) On the other hand, a non-convex auto-term support produces cross-terms, inner interference terms, in the time-frequency plane. (c) Multi-component signals lead to outer interference terms that are due to the interaction between different auto-terms in the time-frequency plane. 
kernels. However, design of a single kernel for the entire signal may lead to some compromises when analysing multi-component signals (Jones and Baraniuk, 1995). The adaptation of the kernel at each time to achieve optimal local performance usually provides better TFDs at the expense of significantly increased computational complexity (Jones and Baraniuk, 1995). Nevertheless, the design of a single kernel at each time instant may lead to similar compromises as in ORGK when there are signal components that overlap in time.

This paper presents a new technique, TFCA, that provides a high-resolution time-frequency characterization of localized signal components (Arıkan et al., 2003; Özdemir and Arıkan, 2000, 2001; Özdemir et al., 2001; Özdemir, 2003). The only assumption made about the components of the signal is that they have non-overlapping supports in the time-frequency plane. As explained in Section 2.4.2, this assumption on the signal components can be relaxed as well. Under the assumption of non-overlapping signal components, the TFCA technique makes use of a component adaptive time warping operation to transform analysed signal components with non-convex supports into ones with convex supports. The warped signal components are extracted by using a time-frequency domain incision algorithm and their corresponding distributions are computed by using directionally smoothed Wigner analysis. The idea is that, for signals with convex supports Wigner distribution provides superior time-frequency resolution with negligible cross-term interference. Finally, by using an inverse warping transformation, the cross-term free distribution of the original, i.e. unwarped, components are obtained. In TFCA, after a component is extracted and its distribution is computed, that component is subtracted from the analysed signal and the same analysis is conducted on the residual signal until distributions for all components are obtained.

One of the contributions of this paper is introduction of warping transformation into time-frequency analysis of ERP signals. As detailed, the warping function is computed by using short-time Fourier transformation, which provides a coarse but cross-term free distribution. Then, the support of the analysed signal component is isolated by using an image segmentation algorithm. After the orientation of the isolated support is identified, time-frequency domain rotations and translations (enabled by fractional Fourier transformation, time shifts and frequency modulations, respectively) are utilized to obtain a support which has a positive and singlevalued spine. Finally, the warping function corresponding to estimated spine is computed by quadrature techniques. Hence, in TFCA, it is assumed that the signal components of the brain have localized time-frequency supports whose corresponding spines can be transformed into positive and single-valued spines by using time-frequency domain rotations and translations.

In contrast to Wigner distribution and its smoothed versions, TFCA yields negligible cross-term cluttering between the different components in the composite signal (outer interference terms) and within the component itself (inner interference terms), while preserving the time-frequency localization of the auto-components. As ERPs have localized time-frequency supports, the TFCA technique may be an appropriate tool for high-resolution ERP analysis. It may provide both an accurate time domain identification and representation of the frequency components that constitute the ERP. TFCA can also extract individual signal components from noisy recordings.

The aim of the present study has been to describe the TFCA technique, and to test its applicability to time-frequency analysis of ERP signals. The technique was tested on a simulated signal and on ERPs that were obtained under the active oddball (OB) paradigm (Sutton et al., 1965). Since the ERP components and also the ERO components that form the OB waveform have been well established (BaşarEroğlu et al., 1992; Polich and Kok, 1995; Karakaş et al., 2000a, 2000b), ERP of OB is an appropriate signal for testing the utility of a signal analysis technique, and for demonstrating the advantages that the technique may possess over others currently used, and cited in the literature. The present study compared the findings that were obtained with TFCA to those obtained with the commonly used time-frequency technique, the Wigner analysis.

\section{Methods and materials}

\subsection{Subjects}

The data were acquired from 20 young volunteering adults (18-29 years; 5 males and 15 females) who were recruited from the university student population. Subjects were naive to electrophysiological studies. Only those individuals who reported being free of neurological or psychiatric problems were accepted. Individuals who were, at the time of testing, under medication that would have affected cognitive processes or who stopped taking such medication, were excluded. The hearing level of the potential subjects was assessed through computerized audiometric testing prior to the experimental procedures. Individuals with hearing deficits were not included in the study, either.

\subsection{Stimuli and paradigms}

The auditory stimuli had $10 \mathrm{~ms} \mathrm{r} / \mathrm{f}$ time, $50 \mathrm{~ms}$ duration and were presented over the headphones at $65 \mathrm{~dB}$ SPL. The deviant stimuli $(n=30-33,2000 \mathrm{~Hz})$ occurred randomly with a probability of about 0.20 within a series of standard stimuli $(n=120-130,1000 \mathrm{~Hz})$ that were presented with a probability of about 0.80 . According to the procedures of the oddball paradigm, participants had to mentally count the occurrence of deviant stimuli and to report them after the session had been terminated (for details of the methodology, see Karakaş et al., 2000a). 


\subsection{Electrophysiological procedures}

Electrical activity of the brain, the prestimulus electroencephalogram (EEG) and the poststimulus ERP, were recorded in an electrically shielded, sound-proof chamber. Recordings were taken from 15 recording sites (ref: linked earlobes; ground: forehead) of the 10-20 system under eyes-open condition. The present study reports findings from the Fz recording site.

Bipolar recordings were made of electro-ocular and electromyographic activity for online rejection (of responses whose amplitudes exceeded $\pm 50 \mu \mathrm{V}$ ) and offline rejection (through visual inspection) of artefacts. Rejection occurred for epochs that contained gross muscular activity, eye-movements or blinks. Electrical activity was amplified and filtered with a bandpass between 0.16 and $70 \mathrm{~Hz}(3 \mathrm{~dB}$ down, $12 \mathrm{~dB}$ /octave). It was recorded with a sampling rate of $500 \mathrm{~Hz}$ and a total recording time of $2048 \mathrm{~ms}, 1024 \mathrm{~ms}$ of which served as the prestimulus baseline. EEG-ERP data acquisition, analysis, and storage were achieved by a commercial system (Brain Data 2.92). A notch filter $(50 \mathrm{~Hz})$ was not activated.

\subsection{Description of TFCA: procedures and applications}

In this section, TFCA is presented in detail. In Section 2.4.1, some preliminaries on the fractional domain warping transformation are provided. Then, in Section 2.4.2., the analysis of multi-component signals by TFCA is demonstrated. Using simulated data, the performance of TFCA is compared to several other techniques of time-frequency analysis.

\subsubsection{Time-frequency analysis of mono-component signals by fractional domain warping}

Time domain warping is especially useful in processing frequency-modulated signals (Meda, 1980; Brown and Rabiner, 1982; Wulich et al., 1990; Coates and Fitzgerald, 2000). A typical member of this class of signals is of the form of $x(t)=A(t) \mathrm{e}^{\mathrm{j} 2 \pi \varphi(t)}$, where $A(t)$ is the amplitude and $\varphi(t)$ is the phase in Hz. Ideally, the warping function, $\zeta(t)$, for this signal should be chosen as the inverse of its phase, $\zeta(t)=\varphi^{-1}\left(f_{\mathrm{s}} t\right)$, where $f_{\mathrm{s}}>0$ is an arbitrary scaling constant. With this choice, the warped function takes the following form: $x_{\zeta}(t)=A(\zeta(t)) \mathrm{e}^{\mathrm{j} 2 \pi f_{\mathrm{s}} t}$, which is a sinusoidal function at frequency $f_{\mathrm{s}}$ with envelope $A(\zeta(t))$. Consequently, the algorithms designed to operate on sinusoidal signals can be utilized on the warped signal, which has a narrow band $A(\zeta(t))$.

Fractional Fourier transformation (FrFT) is a oneparameter generalization of the ordinary Fourier transform. The $a$ th-order, $x_{a}(t), a \in \mathbb{R},|a| \leq 2$ fractional Fourier transform of a function is defined as (Almeida, 1994)

$x_{a}(t)=\left\{F^{a} x\right\}(t) \triangleq \int_{-\infty}^{\infty} B_{a}\left(t, t^{\prime}\right) x\left(t^{\prime}\right) \mathrm{d} t^{\prime}$, where the kernel of the transformation $B_{a}\left(t, t^{\prime}\right)$ is

$$
\begin{aligned}
B_{a}\left(t, t^{\prime}\right) & =A_{\phi} \exp \left(\mathrm{j} \pi\left(t^{2} \cot \phi-2 t t^{\prime} \csc \phi+t^{\prime 2} \cot \phi\right)\right), \\
A_{\phi} & =\frac{\exp (-\mathrm{j} \pi \operatorname{sgn}(\sin \phi) / 4+\mathrm{j} \phi / 2)}{|\sin \phi|^{1 / 2}}, \quad \phi=a \frac{\pi}{2} .
\end{aligned}
$$

From this definition, it follows that first-order FrFT is the ordinary Fourier transform and zeroth-order FrFT is the function itself. The definition of the FrFT is easily extended to outside the interval $[-2,2]$ by noting that $F^{4 k}$ is the identity operator for any integer $k$ and FrFT is additive in index, i.e. $\left\{F^{a_{1}}\left\{F^{a_{2}} x\right\}\right\}(t)=\left\{F^{a_{1}+a_{2}} x\right\}(t)$.

Fractional domain warping is the generalization of the time domain warping to fractional Fourier transform domains (Özdemir et al., 2001). The warped fractional Fourier transform of a signal $x(t)$ is obtained by replacing the timedependence of its FrFT by a warping function $\zeta(t)$. Thus, if $x(t)$ is the time domain signal with the $a$ th-order FrFT $x_{a}(t)$, then the warped FrFT of the signal is given by

$x_{a, \zeta}(t)=x_{a}(\zeta(t))$,

where $\zeta(t)$ is the warping function associated with $x_{a}(t)$.

In TFCA, high resolution distribution of signal components with non-convex time-frequency support (Fig. 2b) is obtained using adaptively chosen fractional domain warping transformations. For each analysed signal component, the warping function is determined on the basis of the component's spine, defined as the centre of mass along the time-frequency domain support of the signal component. To compute the warping function $\zeta(t)$, a single-valued spine is needed. If the support of the signal component $x(t)$ is as shown in Fig. 2e, its spine is a multiple valued function of time. However, if the support is rotated as shown in Fig. 2f, the spine corresponding to the rotated support becomes a single valued function of time and is identical with the instantaneous frequency. The required time-frequency rotation can be performed by the fractional Fourier transformation (Almeida, 1994).

If the spine of the fractional Fourier transformed signal $x_{a}(t)$ shown in Fig. $2 \mathrm{f}$ is given by $\psi_{a}(t), t_{\mathrm{i}} \leq t \leq t_{\mathrm{f}}$, the inverse of the warping function is computed as (Özdemir and Arıkan, 2000)

$$
\begin{gathered}
\Gamma(t)=\int_{t_{\mathrm{i}}}^{t} \psi_{a}\left(t^{\prime}\right) \mathrm{d} t^{\prime}, \quad t_{\mathrm{i}} \leq t \leq t_{\mathrm{f}}, \\
\zeta^{-1}(t)=\frac{\Gamma(t)}{f_{\psi_{a}}}+t_{\mathrm{i}}, \quad t_{\mathrm{i}} \leq t \leq t_{\mathrm{f}},
\end{gathered}
$$

where $f_{\psi_{a}}$ is the mean of the spine

$f_{\psi_{a}}=\int_{t_{\mathrm{i}}}^{t_{\mathrm{f}}} \psi_{a}\left(t^{\prime}\right) \mathrm{d} t^{\prime} /\left(t_{\mathrm{f}}-t_{\mathrm{i}}\right)$.

With these equations, the warping function $\zeta(t)$ becomes

$\zeta(t)=\Gamma^{-1}\left(f_{\psi_{a}}\left(t-t_{\mathrm{i}}\right)\right), \quad t_{\mathrm{i}} \leq t \leq t_{\mathrm{f}}$.

If the spine $\psi_{a}(t)$ is a strictly positive function of time, $\Gamma(t)$ defined in (7) is a monotonically increasing function of time. 

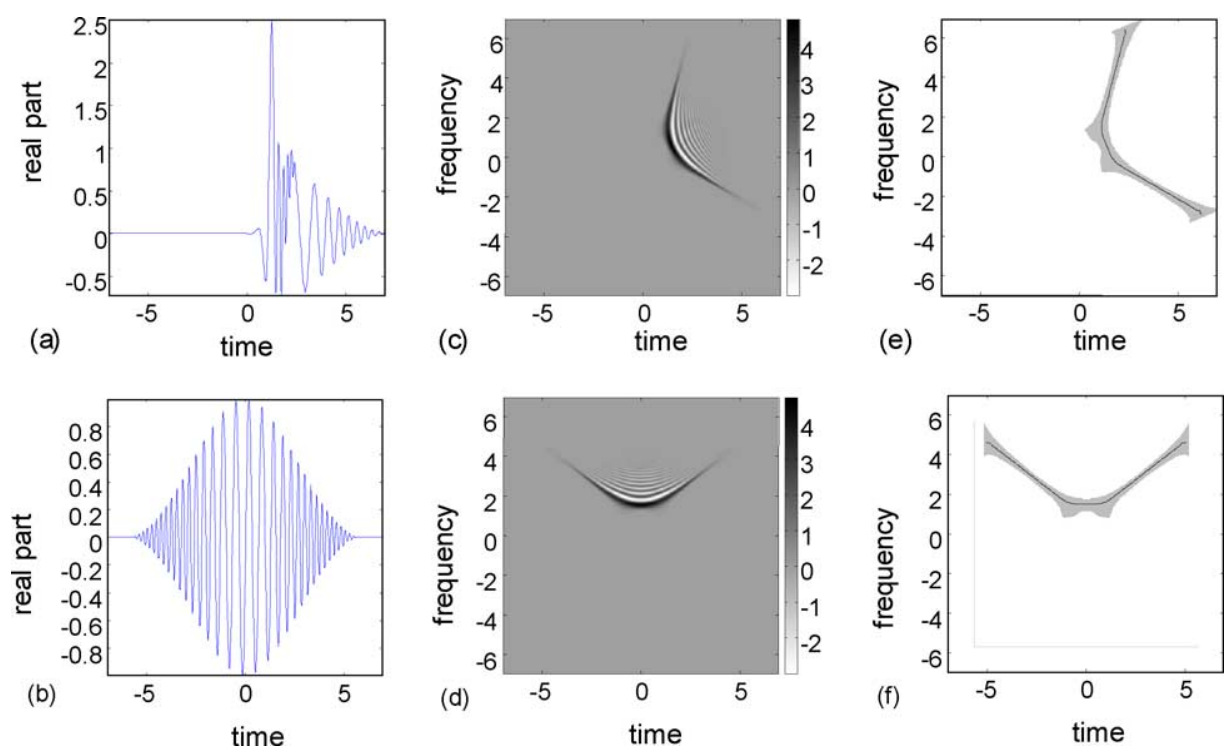

Fig. 2. (a) A signal $x(t)$ and (b) its (-0.75)th-order FrFT $x_{(-0.75)}(t)$; (c) the Wigner distributions of $x(t)$ and (d) $x_{(-0.75)}(t)$; (e) the spines of $x(t)$ and (f) $x_{(-0.75)}(t)$ plotted on the support of their auto-term Wigner distributions. Although the spine in (e) is a multi-valued function of time, the spine corresponding to the rotated support becomes a single-valued function of time as shown in (f).

Therefore, its inverse given in (9) exists and it is unique. Otherwise, the frequency-modulated signal $x_{a}^{\delta_{\mathrm{f}}}(t) \triangleq x_{a}(t) \mathrm{e}^{\mathrm{j} 2 \pi t \delta_{\mathrm{f}}}$ is used, where $\delta_{\mathrm{f}}$ is chosen such that the spine $\psi_{a}^{\delta_{\mathrm{f}}}(t) \triangleq \psi_{a}(t)+$ $\delta_{\mathrm{f}}$ of $x_{a}^{\delta_{\mathrm{f}}}(t)$ is a strictly positive function of time. Hence, for the clarity of the presentation, it will be assumed that $\psi_{a}(t)$ is a strictly positive function of time. To illustrate this, the effect of the warping operation on the simulated signal in Fig. 2a is shown in Fig. 3a. In this example, the warped signal $x_{a, \zeta}(t)$ is computed by using (4) and (6) with $a=-0.75$ and $\delta_{\mathrm{f}}=0$.

After the warping operation, time-frequency support of the signal $x_{a, \zeta}(t)$ is localized around the line segment $\left(\bar{t}, f_{\psi_{a}}\right)$, $t_{\mathrm{i}} \leq \bar{t} \leq t_{\mathrm{f}}$, in the time-frequency plane. Thus, by using the warping operation, the signal component with non-convex time-frequency support is transformed to a component with convex support in the time-frequency plane (Özdemir and Arıkan, 2000).

In order to determine the time-frequency representation of the mono-component signal, first, the Wigner distribution of the warped signal is used to calculate a high-resolution time-frequency representation of the signal in the ath fractional domain. Then, this fractional domain representation has to be rotated back in order to obtain the desired time-frequency representation. The mathematical details of these operations are given in (Özdemir and Arıkan, 2000). The resultant TFD of $x(t)$ obtained by fractional domain warping analysis is given in Fig. $3 \mathrm{~b}$.

\subsubsection{Application of TFCA to the analysis of multi-component signals}

In this section, the TFCA and its steps are demonstrated on a three-component signal $s(t)=\sum_{i=1}^{3} s^{i}(t)$, produced by combining the three components in Fig. $4 \mathrm{a}-\mathrm{c}$ with the simulated additive noise $w(t)$ in Fig. $4 \mathrm{~d}$. The mean ratio of the signal-to-noise power spectral densities was chosen to be $5 \mathrm{~dB}$. The noisy signal $x(t)=s(t)+w(t)$ and its Wigner distribution $W_{x}(t, f)$ are shown in Fig. $4 \mathrm{e}$ and $\mathrm{f}$, respectively. The plot of the Wigner distribution clearly exhibits significant cross-terms.
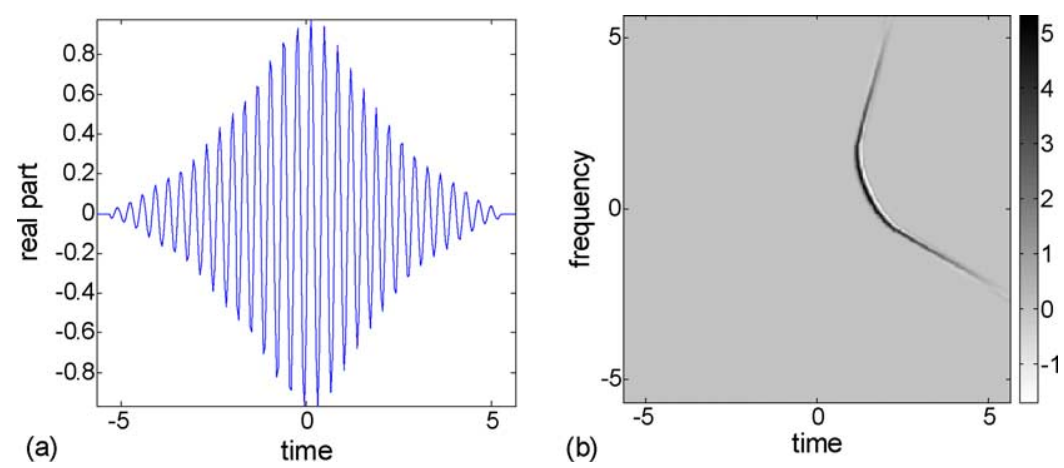

Fig. 3. (a) The warped fractional Fourier transform of the signal in Fig. $2 \mathrm{a}$ and (b) the time-frequency distribution of $x(t)$ obtained by using the fractional domain warping analysis. 

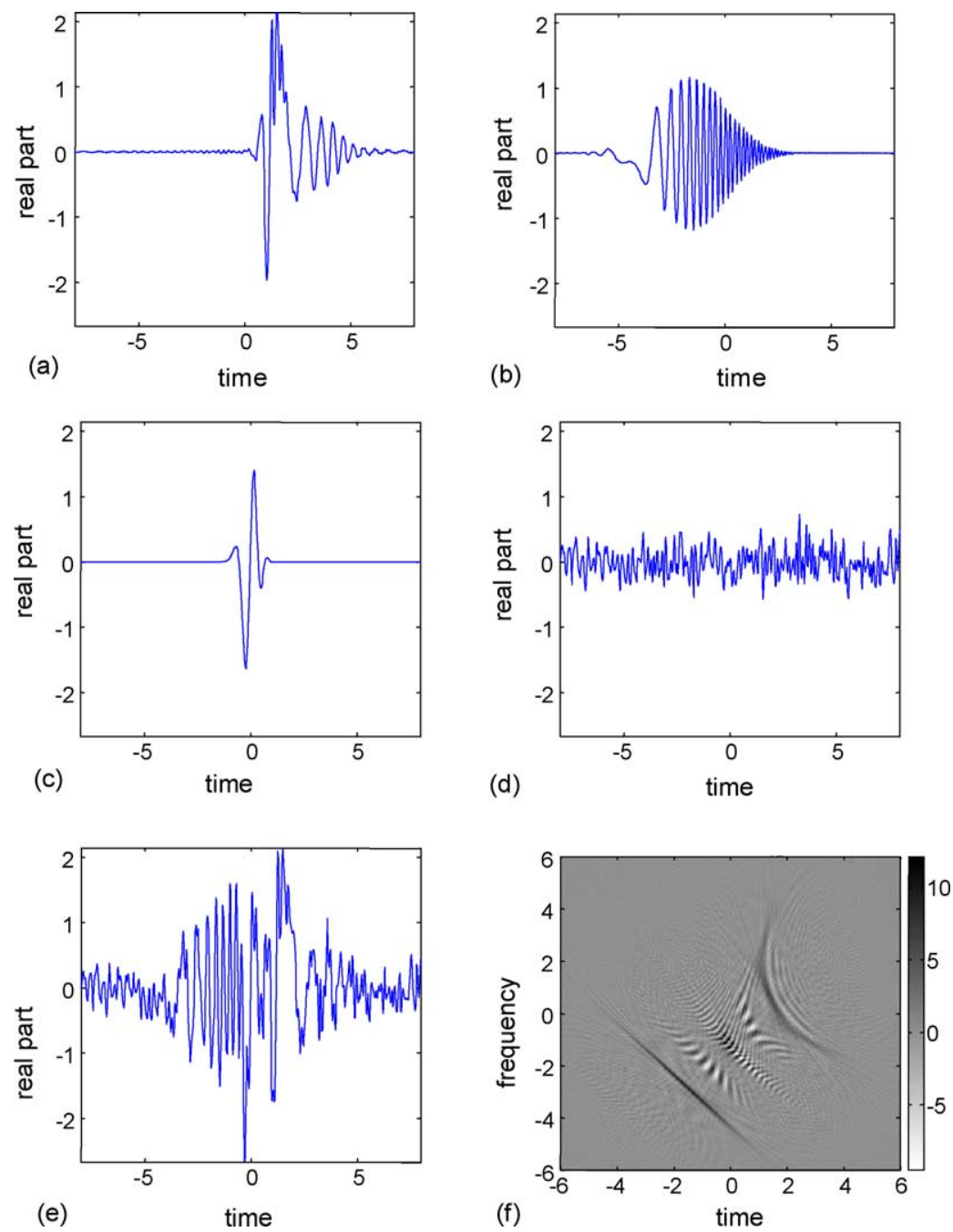

Fig. 4. The three-component signal $x(t)$ shown in (e) is formed by combining the three synthetically generated components given in (a)-(c) with additive noise shown in (d). The Wigner distribution $W_{x}(t, f)$ of the composite signal is given in (f). The signal component in (a) that lies in the upper right part of (f) has a non-convex $t-f$ support, and it suffers from inner interference terms. On the other hand, the component in (c) that lies close to center of (f) is completely masked by the outer interference terms.

The analysis of multi-component signals by TFCA starts with estimating support of the signal in the time-frequency plane. To this end the short-time Fourier transform can be utilized. The advantage in using STFT is that it does not produce cross-term interference, since it is linear, contrary to bilinear time-frequency distributions. On the other hand, STFT has a lower resolution compared to bilinear time-frequency distributions. However, since TFCA uses STFT only to obtain a crude estimate of the signal's support in the time-frequency plane, it may be an acceptable first approach (Durak and Arıkan, 2003). In Fig. 5a, the shorttime Fourier transform, $\operatorname{STFT}_{x}(t, f)$ of the multi-component signal $x(t)$ is shown where $h(t)=\mathrm{e}^{-\pi t^{2}}$ was used as the window function in computing STFT. Although STFT has lower resolution then the Wigner distribution, the supports of all components can be detected when the watershed segmentation algorithm is used (Vincent and Soille, 1991) as shown in Fig. 5b.
In the second stage of TFCA, a component to be analysed by TFCA is chosen as the component where the outer interference term contamination is lower. In the presented example, this component could be either of the two components lying in the lower left part and upper right part of the $t-f$ plane, respectively, as shown in Fig. 5b. In order to present all steps of TFCA in detail, we chose, in this example, the first component $s^{1}(t)$ to be analysed by TFCA as the one that lay in the upper right part of the $t-f$ plane. It had a non-convex $t-f$ support.

Having thus chosen the first component, the appropriate FrFT of order $a_{1}$ was chosen. As discussed in Section 2.4.1, a single valued spine is needed to transform the nonconvex support into a convex one. Thus, the order $a_{1}$ of the FrFT is chosen such that after $a_{1} \pi / 2$ radians rotation of the time-frequency support of $x(t)$ in the clock-wise direction, the spine of the analysed component becomes a single valued function of time. In the example, $a_{1}=-0.75$ was chosen. 

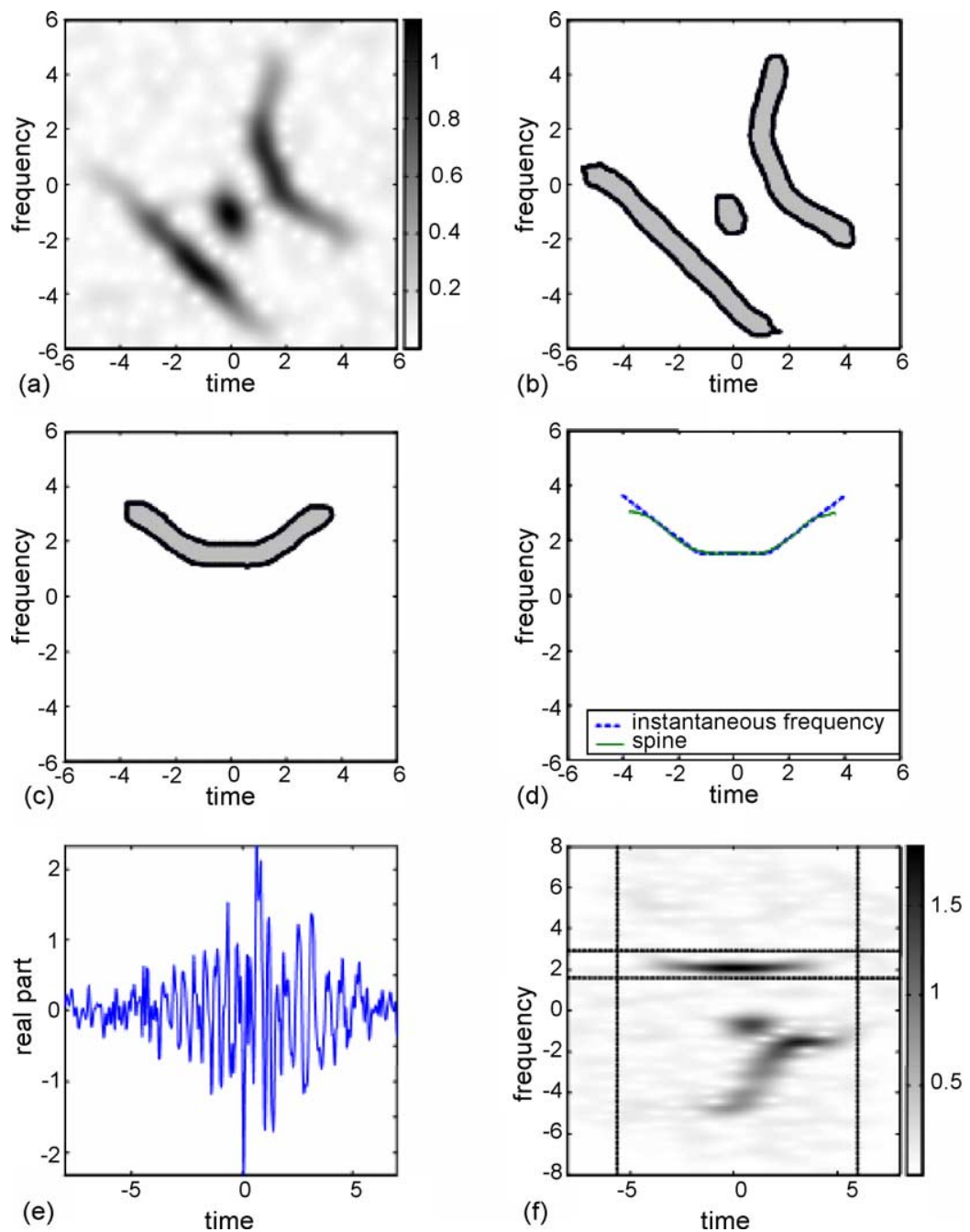

Fig. 5. (a) The short-time Fourier transform of $x(t)$ in Fig. 4e computed by using the window function $h(t)=\mathrm{e}^{-\pi t^{2}}$; (b) supports of the components in STFT computed by using the watershed segmentation algorithm (Vincent and Soille, 1991); (c) the indicator function $M_{a_{1}}(t, f), a_{1}=-0.75$, of the support of the component $s_{1}(t)$ in the $\left(a_{1}\right)$ th fractional domain; (d) the computed spine and the actual instantaneous frequency of the component $s_{1}(t)$ in the $\left(a_{1}\right)$ th fractional domain; (e) the warped FrFT $x_{\left(a_{1}, \varsigma_{1}\right)}(t)$ of the signal in Fig. 4e; and (f) its short-time Fourier transform $\operatorname{STFT}_{x_{\left(a_{1}, \varsigma_{1}\right)}}(t, f)$. The horizontal and vertical lines in (f) outline the supports of the frequency and time domain incision masks, respectively, which are utilized by TFCA to extract the signal component that is located inside the dashed rectangular box.

Actually, any $a_{1}$ in the interval of $[-0.50,-1.00]$ could have reliably been used for this purpose. Note that in the case of signal components with overlapping time-frequency supports, such as two crossing chirp components with one increasing in frequency and the other decreasing in frequency, it may not be possible to obtain a single-valued spine. In such a case, first the overlapping signal components should be extracted from the composite signal. To this purpose, the techniques such as those in McHale and Boudreaux-Bartels (1993) and Hlawatsch et al. (1994), which can synthesize signals from partially known, i.e. non-overlapping part of Wigner domain information, can be used. In these techniques, the optimal signal that best fits to a given Wigner distribution with don't care regions is obtained. Once, such a signal extraction technique is used, the identified signal component can be synthesized even if its Wigner distribution cannot be specified over the region of overlap. Then, the synthesized signal component is subtracted from the signal and TFCA technique proceeds as detailed before for non-overlapping signal components. A detailed study and automatization of such an approach shall be the subject for future work.

In the next stage of the TFCA, the spine $\psi_{a_{1}}(t)$ of the first component $s_{a_{1}}^{1}(t)$ in the domain of the fractional Fourier transforms is estimated. Since after the rotation, the spine of $s_{a_{1}}^{1}(t)$ becomes a single valued function of time, an instantaneous frequency estimation algorithm (Boashash and O'Shea, 1993; Cohen, 1995; Katkovnik and Stankovic, 1998; Baraniuk et al., 2001; Kwok and Jones, 2000) can be used to determine the spine. In this paper, the spine is obtained as

$\psi_{a_{1}}(t)=\frac{\int_{-\infty}^{\infty} f\left|\operatorname{STFT}_{x_{a_{1}}}(t, f) M_{a_{1}}(t, f)\right|^{2} \mathrm{~d} f}{\int_{-\infty}^{\infty}\left|\operatorname{STFT}_{x_{a_{1}}}(t, f) M_{a_{1}}(t, f)\right|^{2} \mathrm{~d} f}$, 
where the magnitude squared STFT is called spectrogram, which is a smoothed bilinear $t-f$ distribution (Cohen, 1995) and the mask $M_{a_{1}}(t, f)$ is the indicator function of the support of $s_{a_{1}}^{1}(t)$, which was obtained automatically using watershed segmentation algorithm (Vincent and Soille, 1991). In the presented example, the estimate of the spine $\psi_{a_{1}}(t)$, computed by using the indicator function $M_{a_{1}}(t, f)$ in Fig. 5c, was obtained as shown in Fig. 5d. In this example, the corresponding root mean square estimation error for the spine was $0.102 \mathrm{~Hz}$. Then, the warped FrFT in $x_{\left(a_{1}, \zeta_{1}\right)}(t)$ Fig. 5e was computed. In order to determine the support of the first warped component, the short-time Fourier transform $\operatorname{STTF}_{x\left(a_{1}, \zeta_{1}\right)}(t, f)$ of the warped signal, was calculated (Fig. 5f). The STFT component with convex support corresponds to the first warped component. Note that in the computation of the STFT, a Gaussian window, $h(t)=\mathrm{e}^{-\pi t^{2} / 4}$, was used.

The next stage of processing involved the extraction of the warped signal component. For this purpose, various time-frequency processing techniques (e.g. Hlawatsch et al., 1994, 2000; Erden et al., 1999; Hlawatsch and Kozek, 1994; McHale and Boudreaux-Bartels, 1993; Boudreaux-Bartels and Parks, 1986) can be used. In the following, results based on the time-frequency domain incision technique (Erden et al., 1999) will be presented. The warped signal component could be extracted by using a simple incision technique by first applying a frequency domain mask $H_{1}(f)$ to $S(f)$ and then a time domain mask $h_{2}(t)$ to the result of the first step. To determine the supports of the frequency and time domain masks, first, the support of the warped signal component was automatically computed by using the watershed segmentation algorithm. Then, the supports of the masks were chosen such as to enclose the support of the first component in $\operatorname{STFT}_{x_{\left(a_{1}, \zeta_{1}\right)}}(t, f)$ into the rectangular region between the horizontal and vertical dashed lines (Fig. 5f). In this way, the time-frequency support of the estimated signal component was bounded by the dashed-box around this component. Formally, the warped component estimate was obtained as

$\hat{s}_{a_{1}, \zeta_{1}}^{1}(t)=h_{2}(t)\left[h_{1}(t) * x_{a_{1}, \zeta_{1}}(t)\right]$,

where $h_{2}(t)$ is the time domain mask, $h_{1}(t)$ is the inverse Fourier transform of the frequency domain mask $H_{1}(f)$, and * denotes the convolution operation. Having obtained an estimate for $s_{a_{1}, \zeta_{1}}^{1}(t)$, an estimate of $s^{1}(t)$ could easily be computed by inverse warping, and inverse fractional Fourier transformation operations, respectively

$\hat{s}_{a_{1}}^{1}(t)=\hat{s}_{a_{1}, \zeta_{1}}^{1}\left(\zeta_{1}^{-1}(t)\right), \quad \hat{s}^{1}=F^{\left(-a_{1}\right)} \hat{s}_{a_{1}}^{1}(t)$

In the presented example, the FrFT order is $a_{1}=-0.75$. The resultant signal obtained after these operations is shown in Fig. 6a superimposed by the actual component $s^{1}(t)$ in Fig. 4 a. The good fit between the estimated and actual signals indicates the accuracy of the time-frequency domain incision algorithm despite a high noise level.
After extraction of the first component, the same analysis is repeated on the residual signal $r^{1}(t)=x(t)-\hat{s}^{1}(t)$ in order to estimate the second component and its corresponding TFD. Continuing in this manner, all components of the composite signal will eventually be estimated. In Fig. $6 \mathrm{~d}$ and g, the estimates of the remaining signal components are plotted superimposed by the actual components constituting $x(t)$ from Fig. $4 \mathrm{~b}$ and c, respectively. As the plots show, TFCA provided quite accurate estimates of the actual signal components in this simulation example.

Before comparing the performance of TFCA with some well-known time-frequency analysis techniques, it should be noted that, if the identified support of the warped signal component is free of outer interference terms, the TFCA can determine the time-frequency distribution of that component without the use of signal extraction. Otherwise, the signal components that have outer interference terms can only be analysed reliably after the extraction of those signal components that cause the interference. The extraction of signal components is a must in this case. Since TFCA aims not only to determine the time-frequency distribution, but also to extract the identified signal components, signal extraction is always an integral part of TFCA.

Once the TFCA isolates the individual signal components, their corresponding high-resolution time-frequency representations could be obtained as described in Section 2.4.1 for mono-component signals. The TFDs of the individual components are displayed in Fig. 6b, e and h, respectively. TFCA then computed the time-frequency distribution of the composite signal by summation of the computed time-frequency distributions of the individual components as shown in Fig. 7b. As the figure clearly shows, the computed distribution has a very sharp resolution and negligible outer or inner interference terms.

Fig. $6 \mathrm{c}, \mathrm{f}$ and i demonstrate the application of WA to the composite signal in Fig. $4 \mathrm{e}$ to the estimation of the signal components in Fig. 6a, d, and g. Using quadratic B-splines as basis for WA, the composite signal was sampled at $16 \mathrm{~Hz}$ and decomposed into wavelet coefficients up to the third level. From the coefficients of the wavelet decomposition, the corresponding responses were recovered for the frequency intervals [2,4], [1,2], [0,1] Hz (Fig. 6c, f and i). In this simulation scenario, the wavelet transform failed to yield the components of the simulated signal in Fig. 6a, d, and g (cf. also Fig. 4a-c). This happened because the components of the simulated signal were not localized in the frequency intervals determined by wavelet transform, which uses fixed basis functions.

In order to asses the performance of TFCA qualitatively, the auto-term Wigner distribution in Fig. 7a may be utilized. As shown in this figure, the auto-term Wigner distribution has no cross-term interference and it has a very high auto-term concentration. It is therefore reasonable to expect that a good time-frequency analysis algorithm yield a time-frequency distribution close to the auto-term Wigner distribution. Indeed, a comparison of Fig. 7a and b shows that there is a good fit between the auto-term Wigner distribution and TFD 

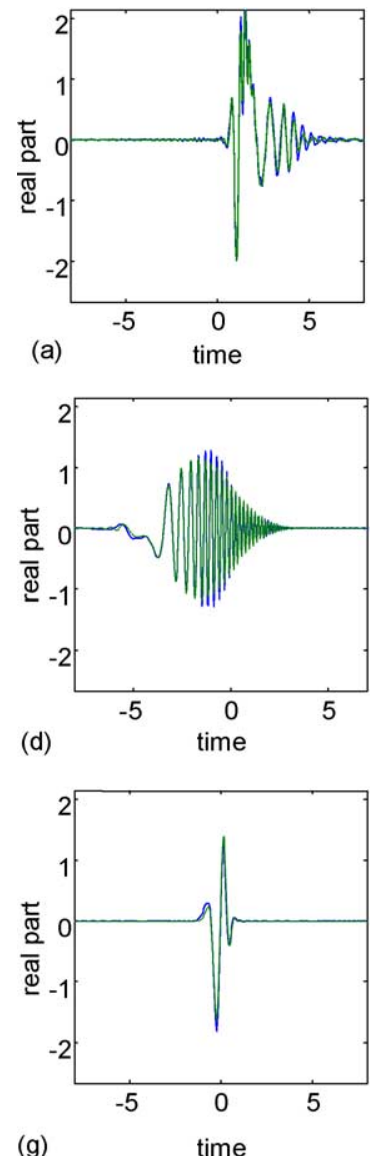
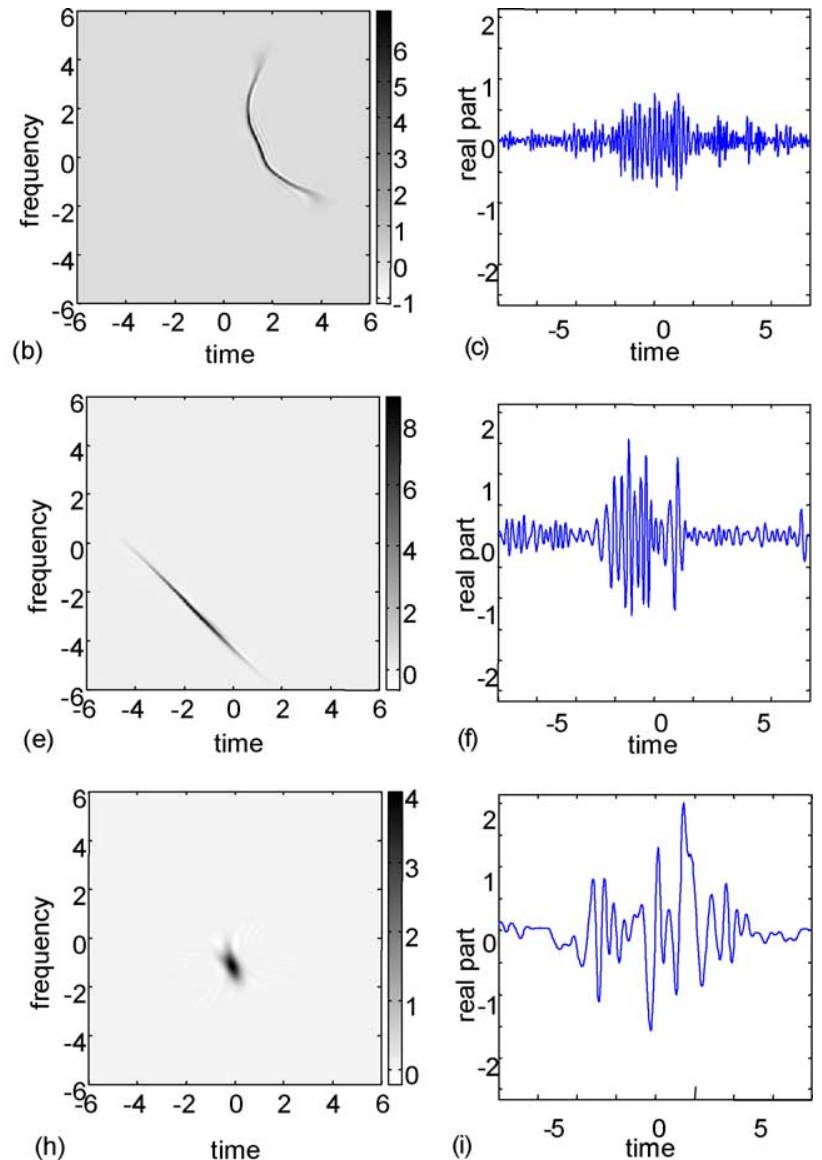

Fig. 6. Parts (a), (d) and (g) are the same components as in Fig. 4a-c, together with their estimates. In these plots, the estimated components are superimposed by the actual components to show the performance of TFCA at a high noise level. Parts (b), (e) and (h) show the TFDs of the respective components obtained with TFCA. The components of the composite signal in Fig. 4e are also estimated by using a wavelet decomposition of order 3 . The signal details D2 and D3 in (c) and (f), and the approximation signal in (i) do not resemble the actual signal components in Fig. 4a-c, respectively. Hence, as this example shows, the wavelet analysis may fail to recover the actual signal components, since the wavelet transform uses fixed basis functions.

obtained with TFCA. It should however be noted that this type of comparison is only possible for simulated signals since the auto-term Wigner distribution can only be computed for a limited set of simulated signals but not for real ERP signals.

The auto-term Wigner distribution plotted in Fig. 7a also provides a clue of the low performance of the wavelet analysis when applied to simulated signals. As it can be seen in the auto-term Wigner distribution in Fig. 7a, all three signal components have considerable energy in the frequency intervals $[2,4],[1,2]$ and $[0,1] \mathrm{Hz}^{2}$ recovered by the wavelet analysis. It should therefore not be surprising that the wavelet analysis could not identify any of the three signal components in Fig. 6 as single entities, and that the recovered frequency bands did not provide accurate estimates of the actual signal components. These findings clearly demonstrate that, if a fixed wavelet basis and frequency intervals are used in the analysis of signals whose components overlap in frequency, the wavelet analysis fails to identify the signal components and to extract them.

\footnotetext{
${ }^{2}$ Note that, if the frequency interval $\left[f_{a}, f_{b}\right]$ is chosen, the wavelet analysis recovers the frequency interval $\left[f_{a}, f_{b}\right] \cup\left[-f_{b},-f_{a}\right]$.
}

The performance of TFCA was compared with that of the smoothed pseudo-Wigner distribution (Fig. 7c) and the well known data-adaptive technique, the optimal radially Gaussian kernel TFD technique (Baraniuk and Jones, 1993) (Fig. 7d). If the smoothing of the Wigner distribution cannot sufficiently suppress the cross-terms, cross-terms remain in the resulting TFD. Otherwise, the auto-term concentration degrades considerably. In Fig. 7d, the result for ORGK time-frequency distribution is given at a volume parameter $\alpha=3$. Although ORGK is able to resolve all three components, there is significant cross-term interference in the arising TFD. Furthermore, there is a distortion in the auto-term of the component with non-convex $t-f$ support. A quantitative comparison of TFCA, and other TFDs can be found in Özdemir (2003). The steps of the implementation of TFCA can be summarized as Algorithm 1.

Algorithm 1. Steps of the time-frequency component analyser.

Purpose of the algorithm: Given a multi-component sampled signal $x\left(n / \Delta_{x}\right),-N / 2 \leq n \leq N / 2-1$, extract its 

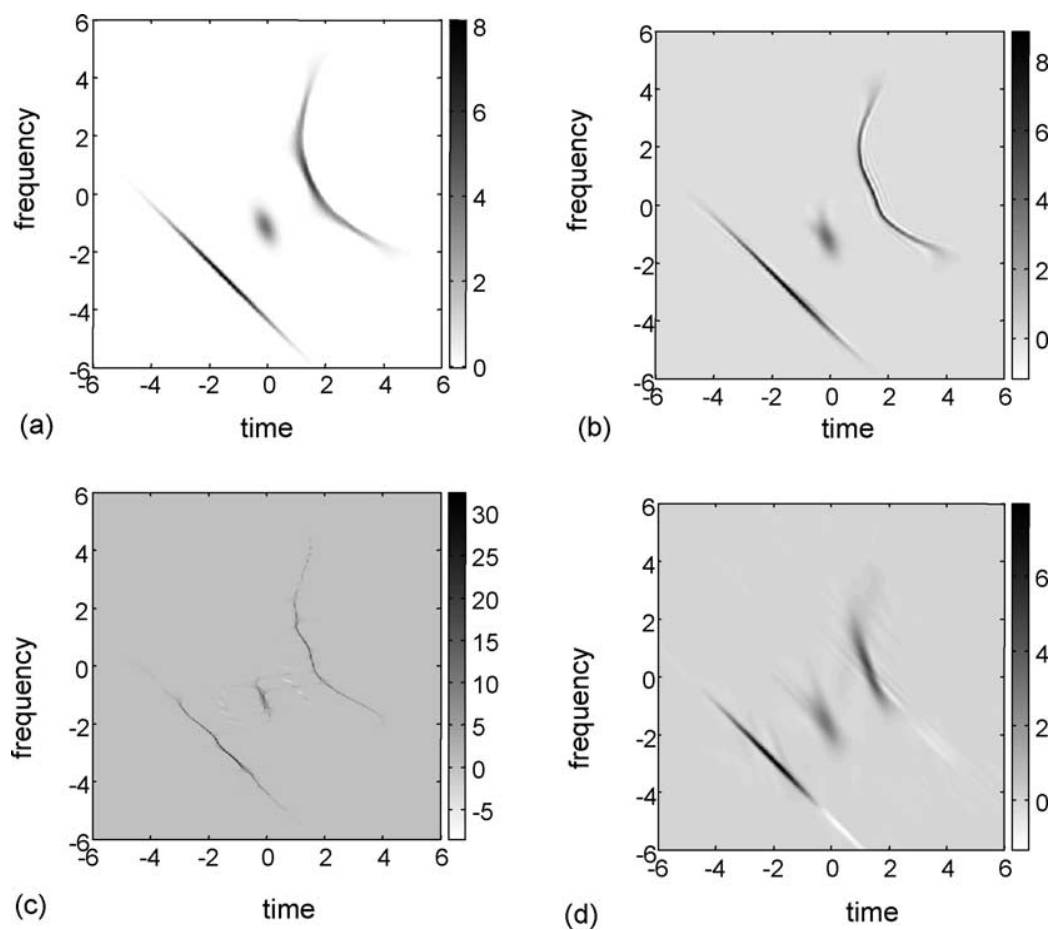

Fig. 7. (a) Auto-term Wigner distribution of the simulated signal in Fig. 4e which was obtained by removing the interference terms from the Wigner distribution in Fig. 4f. Note that, although the auto-term Wigner distribution is a desired distribution, it is, in practice, not computable. It could have been computed in this simulation example, because the simulated components, which constitute the multi-component signal, were available. Parts (b)-(d) show the time-frequency distributions obtained with TFCA, the smoothed pseudo-Wigner distribution and the optimal radially Gaussian kernel time-frequency distribution, respectively. In this example, the volume parameter of ORGK was chosen $\alpha=3$, and respective lengths of the time and frequency smoothing windows for the smoothed pseudo-Wigner distribution were chosen $N / 10$ and $N / 4$, where $N$ was the duration of the sampled analysed signal.

components and compute its time-frequency distribution. It is assumed that $x(t)$ is scaled before its sampling so that its Wigner distribution is inside a circle of a diameter $\Delta_{x} \leq \sqrt{N}$ (see Ozaktas et al., 1996).

\section{Steps of the algorithm:}

1. Initialize the residual signal and the iteration number as $r^{0}(t):=x(t), i:=1$, respectively.

2 . Identify the time-frequency support of the component $s_{i}(t)$ using the watershed segmentation algorithm (Vincent and Soille, 1991). After manually determining the appropriate rotation angle $\phi_{i}$ and the fractional domain $a_{i}=2 \phi_{i} / \pi$, estimate the spine $\psi_{i, a_{i}}(t)$ of the fractional Fourier transform $x_{a_{i}}(t)$ using an instantaneous frequency estimation algorithm. Then, determine the amount of the required frequency shift $\delta_{f_{i}}$ on the spine $\psi_{i, a_{i}}(t)$.

3. Compute the sampled FrFT $r_{a_{i}}^{i-1}(k T), a_{i}=2 \phi_{i} / \pi$, from $r^{i-1}(k T)$ using the fast fractional Fourier transform algorithm (see Ozaktas et al., 1996).

4. Define the warping function $\zeta_{i}(t)=\Gamma_{i}^{-1}\left(f_{\psi_{i}}(t-\right.$ $\left.\left.t_{1}\right)\right)$, where $\Gamma_{i}(t)=\int_{t_{1}}^{t}\left[\psi_{a_{i}}\left(t^{\prime}\right)+\delta_{f_{i}}\right] \mathrm{d} t^{\prime}$ and $f_{\psi_{i}}=$ $\Gamma_{i}\left(t_{N}\right) /\left(t_{N}-t_{1}\right)$. Compute the sampled warping function $\zeta_{\mathrm{i}}(k T)$.
5. Compute the sampled warped signal $r_{a_{i}, \zeta_{i}}^{i-1}(k T)$ as

$$
\begin{aligned}
r_{a_{i}}^{i-1, \delta_{\mathrm{f}_{i}}}(k T) & =\mathrm{e}^{\mathrm{j} 2 \pi \delta_{\mathrm{f}_{i}} k T} r_{a_{i}}^{i-1}(k T), \\
r_{a_{i}, \zeta_{i}}^{i-1, \delta_{\mathrm{f}_{i}}}(k T) & =\mathrm{e}^{-\mathrm{j} 2 \pi \delta_{\mathrm{f}_{i}} k T} r_{a_{i}}^{i-1, \delta_{\mathrm{f}_{i}}}\left(\zeta_{i}(k T)\right) .
\end{aligned}
$$

6. Estimate the $i$ th component by incision of the time-frequency domain as

$\hat{s}_{a_{i}, \zeta_{i}}^{i, \delta_{f_{i}}}(t)=h_{2}(t)\left[h_{1}(t) * r_{a_{i}, \zeta_{i}}^{i-1, \delta_{f_{i}}}(t)\right]$,

where $h_{2}(t)$ is a time-domain mask and $h_{1}(t)$ is the inverse Fourier transform of a frequency domain mask $H_{1}(f)$.

7. For each TFD slice of $s^{i}(t)$, compute $y_{a_{i}, \zeta_{i}}(k T)=$ $\hat{s}_{a_{i}, \zeta_{i}}^{i, \delta_{f_{i}}}(k T) \mathrm{e}^{\mathrm{j} 2 \pi \Delta_{\psi} \zeta_{i}(k T)}$, after choosing the slice offset $\Delta_{\psi}$.

8. Compute the sampled TFD $\mathbb{T F}_{y_{a_{i}, S_{i}}}\left(m \bar{T}, f_{\psi_{i}}\right), t_{1} / \bar{T} \leq$ $m \leq t_{N} / \bar{T}$ of $y_{a_{i}, \zeta_{i}}(t)$ using the directional smoothing algorithm (cf. Özdemir and Arıkan, 2000), where $\bar{T}$ is the sampling interval of the TFD slice.

9. The TFD slice of $s^{i}(t)$ is given by

$\mathbb{T F}_{s^{i}}\left(t_{r}(m \bar{T}), f_{r}(m \bar{T})\right)=\operatorname{TF}_{y_{a}, \zeta}\left(m \bar{T}, f_{\psi}\right)$,

where $\left(t_{r}(m \bar{T}), f_{r}(m \bar{T})\right)$ define a curve in the time-frequency plane parameterized by the variable $m \bar{T}$ 


$$
\begin{aligned}
t_{r}(m \bar{T})= & \zeta(m \bar{T}) \cos \left(\frac{a_{i} \pi}{2}\right)-(\psi(\zeta(m \bar{T})) \\
& \left.+\Delta_{\psi}\right) \sin \left(\frac{a_{i} \pi}{2}\right), \\
f_{r}(m \bar{T})= & \zeta(m \bar{T}) \sin \left(\frac{a_{i} \pi}{2}\right)+(\psi(\zeta(m \bar{T})) \\
& \left.+\Delta_{\psi}\right) \cos \left(\frac{a_{i} \pi}{2}\right), \quad \frac{t_{1}}{\bar{T}} \leq m \leq \frac{t_{N}}{\bar{T}} .
\end{aligned}
$$

10. Estimate the sampled $s^{i}(t)$ by taking the inverse of the warping, frequency modulation and the fractional Fourier transformation on the sampled $\hat{s}_{a_{i}, \zeta_{i}}^{\delta_{f}}(t)$

$$
\begin{aligned}
& \hat{s}_{a}^{i, \delta_{\mathrm{f}_{i}}}(k T)=\mathrm{e}^{\mathrm{j} 2 \pi \delta_{\mathrm{f}_{i}} \zeta_{i}^{-1}(k T)} \hat{s}_{a_{i}, \zeta_{i}}^{i, \delta_{\mathrm{f}_{i}}}\left(\zeta_{i}^{-1}(k T)\right), \\
& \hat{s}_{a_{i}}^{i}(k T)=\mathrm{e}^{-\mathrm{j} 2 \pi \delta_{\mathrm{f}_{i}} k T} \hat{s}_{a_{i}}^{i, \delta_{\mathrm{f}_{i}}}(k T), \\
& \hat{s}^{i}(k T)=\left\{F^{\left(-a_{i}\right)} \hat{s}_{a_{i}}^{i}\right\}(k T) .
\end{aligned}
$$

11. Compute the residual signal $r^{i}(k T)=r^{i-1}(k T)-$ $\hat{s}^{i}(k T)$.

if any signal component is left in residual signal $r^{i}(k T)$ then
Set $i=i+1$, and GOTO step 2, else

Compute the $t-f$ distribution of the composite signal as the sum of the $t-f$ distributions of individual signal components.

\section{endif}

\section{Results}

Fig. 8 shows the results of the TFCA analysis of the averaged ERP (Fig. 8a) of a trial subject ("GUOZ"). The ERP was obtained in response to deviant stimuli under the oddball paradigm. The ORGK provided a highly blurred distribution of the ERP components in the time-frequency plane (Fig. 8b). TFCA showed that the ERP was composed of one prestimulus (component 1) and four poststimulus (components 2-5) signal components (Fig. 8c, e, g, i and k) and these were clearly and sharply localized in the time-frequency plane (Fig. 8d, f, $\mathrm{h}, \mathrm{j}$ and $\mathrm{l}$ ). The high amplitude components 2 and 3 along with component 4 contributed to the P300 component of the time

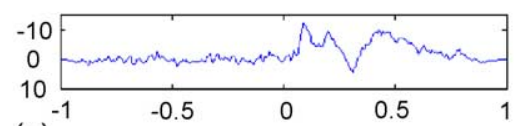

(a)
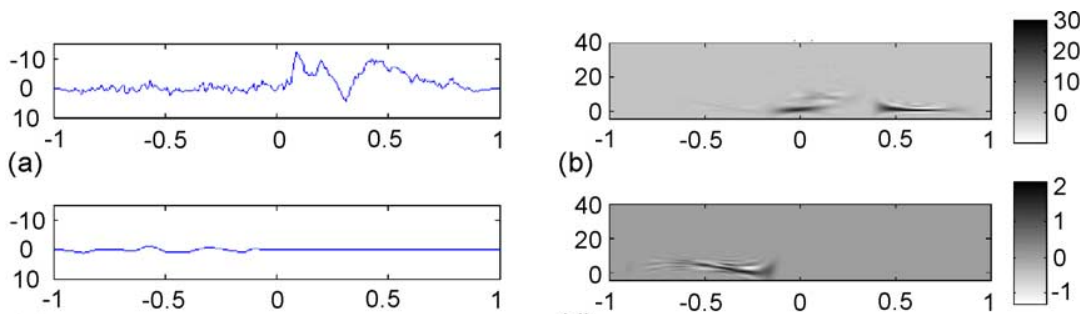

(c)

(b)
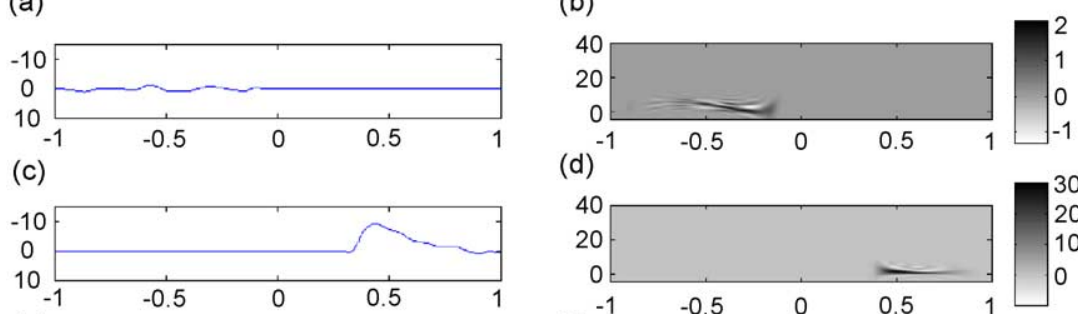

(e)

(d)

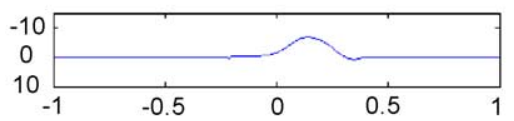

(g)
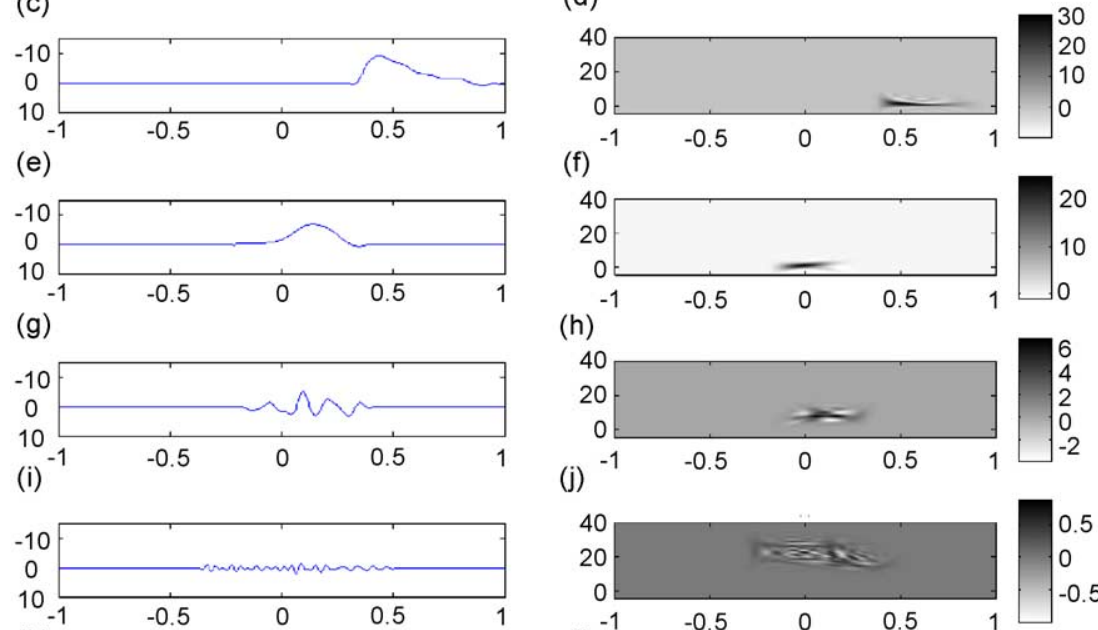

(k)

(h)

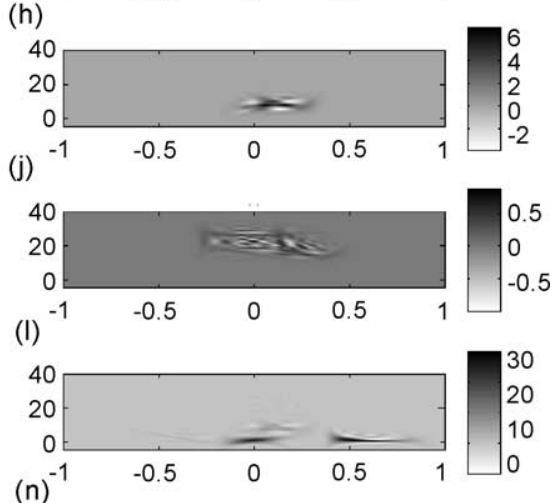

Fig. 8. TFCA analysis of the average ERP evoked by deviant stimuli under the OB paradigm in a trial subject ("GUOZ”). Right axes (b, d, f, h, j and 1): frequency in Hz. Note that the individual time-frequency representations have scales proportional to the strength of the corresponding component. (a) Original ERP; (b) its ORGK TFD; (c, e, g, i and k) time domain representations of ERP components obtained with TFCA; (d, f, h, j and l) corresponding components $(1-5)$ in the time-frequency distributions; (m) absolute value of the difference between the reconstructed superposition and the original ERP given in (a); (n) superposition of the extracted time-frequency representations. 
domain. Component 3 also formed the general waveform of the early negative complex in the ERP waveform. Taking the central value into account, components 2 and 3 were basically due to the delta frequency. However, there were transitions to neighboring frequencies such that components 2 and 3 also included the theta frequency. Component 4 contributed to N100 and N200 in the ERP waveform. Concerning the frequency, component 4 covered basically the theta but also the alpha frequencies. Component 5 was the smallest both in amplitude and energy and it was due to the beta oscillation. It contributed to the early N100 and N200 peaks on the ERP waveform. The mean amplitude of the residual which was obtained by subtracting the reconstructed ERP from the recorded ERP was of the order of $0.6 \mu \mathrm{V}$ (Fig. 8m). This indicated that composite TFCA (Fig. 8n) yielded an accurate decomposition of the ERP.

Fig. 9 demonstrates the inter-subject stability of components produced by TFCA. Fig. 9a presents the time domain grand (ensemble) average ERP waveform computed from the individual responses (508 sweeps from 20 subjects) in response to deviant stimuli under the OB paradigm and Fig. 9b presents the composite distribution of components produced by TFCA. According to TFCA, the grand average ERP was composed of three poststimulus signal components and these were clearly and sharply localized in the time-frequency plane. The high amplitude components 1 and 2 (due basically to delta but also to the theta frequency range) along with component 3 (due basically to theta but also to the alpha fre- quency range) contributed to the P300. Component 2 helped shape the waveform of the early negative complex and component 3 produced N100 and N200 components. When the reconstructed waveform, the sum of the components that were obtained with TFCA, was subtracted from the grand average ERP, the residual signal again had a very small mean amplitude of the order of $0.2 \mu \mathrm{V}$. Fig. 9c, e and g each present the ERP waveform of a different subject; Fig. 9d, f and h present the distribution of the respective TFCA components for these subjects. Fig. 9 shows that the time-frequency distribution of the TFCA components are similar across single-trial subjects and also are well represented by the distribution for the grand average ERP.

Fig. 10 allows an intra-subject ("FEBE") comparison of the distribution of TFCA components for three successive portions (1-30, 31-60 and 61-100\%) of the total number of epochs. Fig. 10a-c shows the average ERP waveforms for the trial subject for the three successive portions of the recording period. Each portion of epochs yielded similar poststimulus components (Fig. $10 \mathrm{~g}-1$ ). There was a high amplitude component in the delta frequency range: this was component 2 in all recordings. Another component was in the theta frequency range: In all epochs, this was component 3. The time-frequency distribution of the components in the composite TFCA are given in Fig. 10m-o. The residuals in Fig. 10p, $r$ and s are of the order of $3 \mu \mathrm{V}$, indicating that TFCA yielded an accurate decomposition of the ERP. The value is higher than that calculated for the total number of sweeps

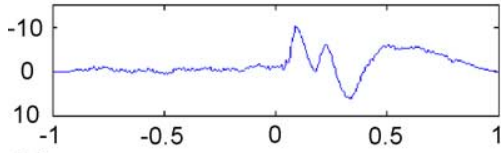

(a)

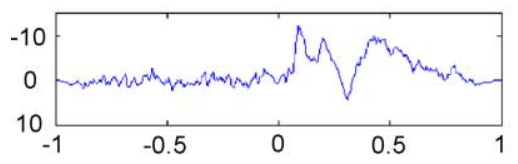

(c)

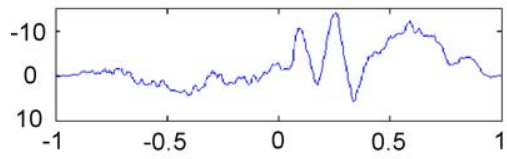

(e)

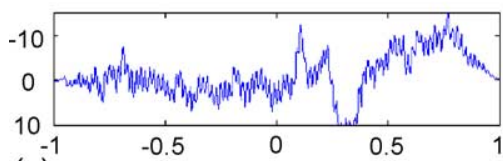

(g)

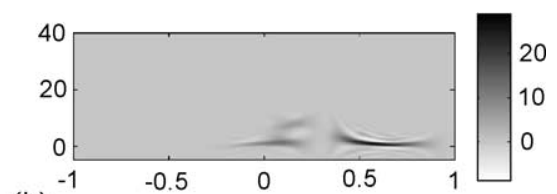

(b)

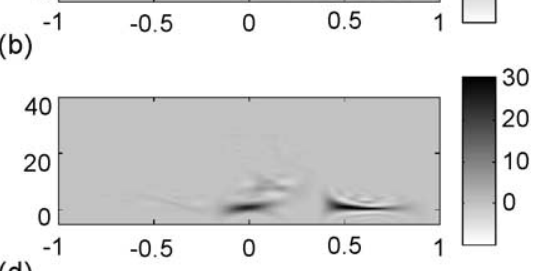

(d)

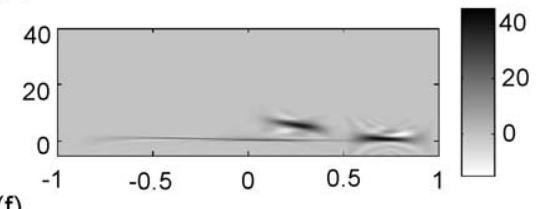

(f)

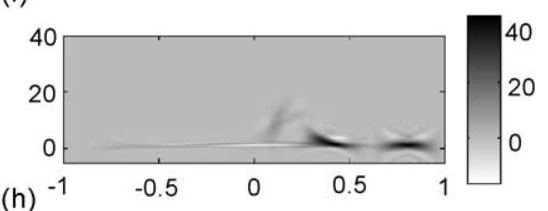

Fig. 9. TFCA analysis of the grand average ERP and averages for single-trial subjects ("GUOZ", "FEBE" and "GOOZ") evoked by deviant stimuli under the OB paradigm. Right axes (b, d, f and h): frequency in Hz. Note that the individual time-frequency representations have scales proportional to the largest energy component corresponding to each subject. (a and b) Grand average ERP and the composite time-frequency representation produced by TFCA; (c, e and g) ERP averages for single-trial subjects ("GUOZ", "FEBE" and "GOOZ"); ( $\mathrm{d}, \mathrm{f}$ and $\mathrm{h}$ ) the composite time-frequency representations for each ERP average produced by TFCA. 


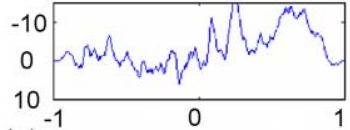

(a)

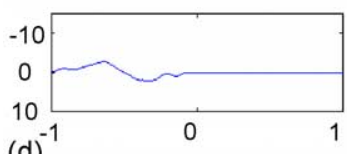

(d)

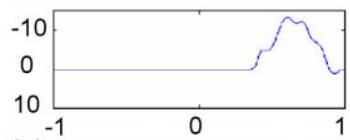

(g)

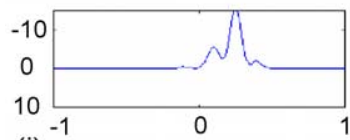

(j)

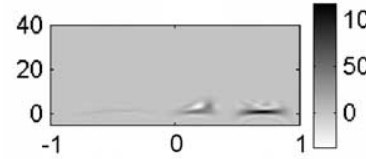

(m)

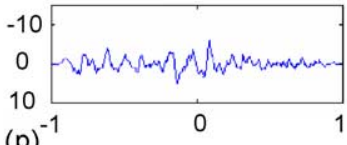

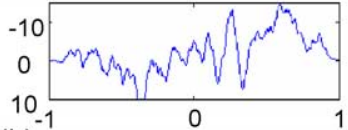

(b)

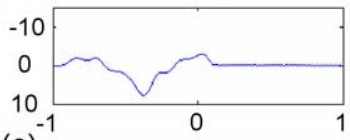

(e)

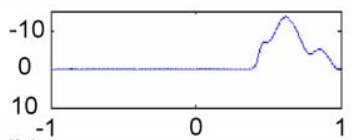

(h)

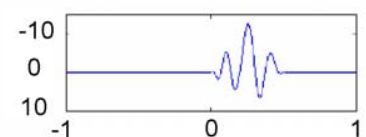

(k)

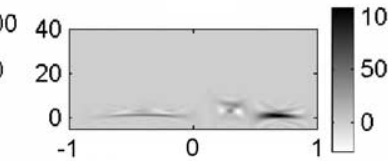

(n)

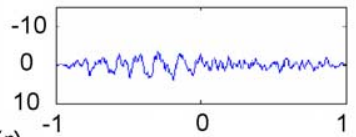

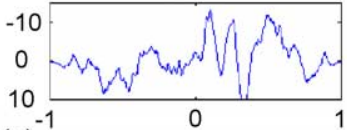

(c)

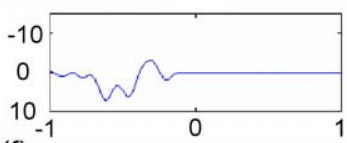

(f)

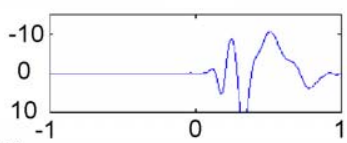

(i)

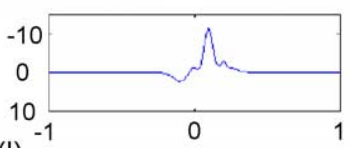

(I)

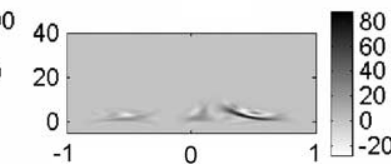

(o)

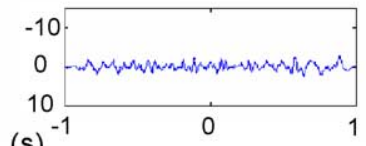

(s)

Fig. 10. TFCA analysis of ERPs of a single-trial subject ("FEBE") averaged for the three successive portions of the recording period. Right axes (m-o): frequency in Hz. (a-c) Original ERPs; (d-1) time domain representations of ERP components obtained with TFCA; (m-o) the corresponding composite time-frequency representations produced by TFCA; (,$r$ and s) absolute value of the difference between the reconstructed and the original ERPs given in (a), (b) and (c), respectively.

(for single trial averages: $0.59-0.64 \mu \mathrm{V}$; for grand average: $0.14-0.20 \mu \mathrm{V})$. This would be expected since the total number of sweeps were divided into three, lending a fewer number of sweeps per block for analyses. Overall, Fig. 10 shows that the time-frequency distribution of the TFCA components are similar across the recording period.

\section{Discussion}

The present study applied the TFCA technique with the aim at describing the electrical responses of the brain in the time-frequency plane. This was achieved by the application of fractional Fourier transform, warping and the fractional domain incision, all utilized by the TFCA technique. TFCA suppressed cross-term interference (both inner and outer) and had a high accuracy in auto-term time-frequency representation. Having properties, the TFCA technique can therefore be used for a high-resolution analysis of mono- and multi-component signals with linear or curved time-frequency supports.

\subsection{Comparison of our results with TFCA with those of previous studies on the frequency-domain responses of the brain}

There is an extensive literature of studies on the cognitive psychophysiology of the stimulus-related time signals: the peaks on the ERP waveform (Sutton et al., 1965; Donchin et al., 1986; Donchin and Coles, 1988; Johnson, 1988; BaşarEroğlu et al., 1992; Karakaş, 1997; Karakaş and Başar, 1998; Karakaş et al., 2000a, 2000b). The ERP peaks at a latency around $200 \mathrm{~ms}$ are related to attention: the early N200 to preattention and the late $\mathrm{N} 200$ to focused attention (Naatanen, 1982, 1990, 1992; Ritter et al., 1992; Naatanen et al., 1993; Winkler et al., 1992; Tervaniemi et al., 1994). Accordingly, the overall N200 peak was obtained, in the present study, in a distinct form under the OB paradigm in response to deviant stimuli where trial subjects concentrated on, and counted the stimuli.

The amplitude of the P300 peak represents the allocation of attentional resources (Wickens et al., 1983; Kramer and Strayer, 1988; Humphreys and Kramer, 1994). It is thus closely related to updating of the memory for stimulus 
recognition and working memory (Sutton et al., 1965; Donchin and Coles, 1988; Johnson, 1988; Polich and Margala, 1997). Again, in line with the above findings, the P300 peak was, in the present study, obtained in a distinct form in response to deviant stimuli under the OB paradigm where the trial subjects had to recognize the stimulus, update memory for a correct count of successively appearing stimuli and decide on the response to be made.

The frequency-domain analysis of the waveforms that was demonstrated in AFC showed prominent selectivities for the delta, theta, beta and gamma bands under various cognitive paradigms such as the single stimulus, oddball and mismatch (Karakaş et al., 2000a, 2000b). When ERPs were appropriately filtered with cut-off frequencies determined from the AFC curves, oscillatory activity occurred in each of the specified frequency ranges. Karakaş et al. (2000a, 2000b) investigated the effect of oscillatory responses on the ERP peaks, basically on N200 and P300, under various cognitive paradigms. The findings showed that the amplitudes of the peaks were determined by the type of cognitive paradigm through a combination of a major contribution of delta and a minor contribution of theta oscillations. These findings were statistically confirmed by stepwise multiple regression analysis, the results of which mathematically demonstrated that the ERP components were mainly due to the additive effects of the delta and theta oscillations. The proportion of variance that the regression model explained was in the range of 94-99\% for different stimuli and paradigms.

TFCA, a technique developed specifically for a precise time-and-frequency localization of components, also demonstrated that an enhanced amplitude and energy were obtained for components that were related to the delta and theta frequencies (components 2 and 3 in particular). As reported in Karakaş et al. (2000a, 2000b), the major contribution to P300 was from components in the delta frequency range. However, there was a minor contribution of components in the theta frequency range as well. The situation was reversed for $\mathrm{N} 200$; the components with the slower frequencies formed the general waveform of the early negativity. The discrimination of N100 and N200 peaks was produced by the components dominantly in the theta frequency range.

Recent studies have shown that beta activity should be taken into account, along with the other oscillations, for a better understanding of brain functions. Başar et al. (2003) showed that beta oscillation is an integral part of the process of face recognition, especially the recognition of one's own grandmother in a photograph. Begleiter and colleagues (Porjesz et al., 2002; Rangaswamy et al., 2002, 2004) found the biochemical, and genetic basis, specifically the $\mathrm{GABA}_{\mathrm{A}}$ receptor genes, for beta activity in the EEG at rest. The authors further showed that the power density of beta oscillation was elevated in alcoholics suggesting that this may be the electrophysiological index of imbalance in the excitationinhibition homeostasis in the cortex.

The present study also identified and extracted the beta oscillation in the ERPs evoked by deviant stimuli under the OB paradigm. Beta oscillation in these components contributed to the ERP peaks N100 and N200. These ERP peaks are related to the physical analysis of stimuli and to attentive processes, respectively (Naatanen, 1982, 1990, 1992; Ritter et al., 1992; Naatanen et al., 1993; Winkler et al., 1992; Tervaniemi et al., 1994).

\subsection{Conclusions: comparison of methods of frequency analysis}

The oscillatory responses of the brain have been presented as the 'paradigm change' in brain research. A growing amount of literature shows the explanatory value of these slow-wave events (Sayers et al., 1974; Başar, 1980, 1998, 1999; Mountcastle, 1992; Karakaş and Başar, 1998; Sannita, 2000; Rangaswamy et al., 2002, 2004; Porjesz et al., 2002; Kamarajan et al., 2004):

- Fourier transform, as a technique of frequency analysis, yields the global frequency composition of the analysed signal in the form of amplitude-frequency characteristics. Digital filtering discerns the oscillatory activity over the time axis that is in the conventional range of brain oscillations, or between the adaptively chosen cut-off frequencies, which are determined from the maxima of the AFC. Wavelet analysis determines the time localization of the distinct wavelet basis components.

Accordingly, most of the existing methods of frequency analysis impose windows on the data. Windows in DF are the adaptively chosen cut-off frequencies. Windows in WA are the appropriately chosen mother wavelets. There were no predefined windows or criteria when signals were analysed with TFCA.

- Of the existing signal analysis techniques, only AFC determines directly the frequency components of the signal. However, this technique does not provide any information on the temporal localization of the frequency components. TFCA yields the relevant oscillatory components that are inherent in ERP. Unlike AFC, TFCA could also determine the time domain representation of the components that shape the ERP. Using techniques that could overcome the cross-term interference either between components (outer) or of the component itself (inner), TFCA could sharply localize components both in the time and in the frequency domain with high temporal, and also high frequency resolution.

The amplitude of the residuals is a measure of the goodness of the time-frequency resolution achieved by TFCA. Residuals are left-over signals after the component extractions. In the present study, the residual values were found to be in the range of $0.59-0.64 \mu \mathrm{V}$ for averages from singletrial subjects and in the range of $0.14-0.20 \mu \mathrm{V}$ for the grand average. These negligible values show that the complex waveform was almost completely decomposed by TFCA. Summation of the extracted components could thus restore to the original waveform. 
- The residuals further demonstrate that TFCA identified and extracted all non-negligible components. Amplitudes of oscillatory activity existing outside the time range for a given component was of the same order of the magnitude as that of the residual. Consequently, after the TCFA analysis, no further significant components are to be expected.

- Signal analysis techniques are based on certain assumptions. The assumption of linearity is peculiar to AFC and that of stationarity is peculiar to AFC and nonlinear dynamic metrics. In wavelet analysis, the templates, themselves, constitute a 'hypothetic model'. The assumption of TFCA is that the analysed signals have one or more components with non-overlapping supports in the time-frequency plane and each component can be rotated in time-frequency plane to have single valued spines.

- The components of ERP are the points of maximal amplitudes: the peaks, on the time-varying ERP. In AFC, the components are distinct maxima of specific frequency ranges; in DF, they are time-varying oscillations in specific frequency ranges; and, in WA, time-varying, adaptive frequency templates. Conventional filtering techniques produce oscillatory components that fall within the cut-off frequencies of the filter. These techniques can thus accurately capture a component whose frequency support does not change with time. However, they cannot differentiate between components if more than one component occur in the same frequency range over the time axis (Cook III and Miller, 1992; Farwell et al., 1993; Karakaş and Başar, 1998).

The findings of the present study demonstrated that components do not always obey the conventional limits of the frequency ranges. There are frequency transitions whose components consist of delta and theta, or alpha and beta oscillations. Fig. 11 presents an ERP averaged from responses to deviant stimuli under the OB paradigm in a trial subject. In this figure, component 1 occupies different frequency bands in different time intervals. Part of component 1 , extracted by TFCA, falls into the delta, and part of it into the theta range. Similarly, while the dominant frequency in component 3 is in the alpha, it also contributes to the beta range. Clearly, for non-stationary signals whose components occupy different frequency bands at different times, digital filtering will only filter-in those parts that fall into the frequency band of the filter. In TFCA, on the other hand, the components are obtained in the form of time-frequency localized 'islets'. These islets show, without any predefined windows, the natural time and frequency spread of the components. Hence, TFCA appears to be an appropriate tool for decomposing ERP into a set of superimposed oscillatory components under variable experimental conditions (Başar and Ungan, 1973; Başar, 1980; Karakaş et al., 2000a, 2000b).

Brain neuroelectricity is the result of the temporal and spatial integration of time-varying oscillatory activity of various frequencies. The brain is essentially a nonlinear and non-stationary system. The time-frequency-domain analysis technique, TFCA, does not assume that the brain is either linear or stationary. Yet, TFCA suppresses the cross-terms (both inner and outer interference terms), which are associated with the Wigner distribution. It accurately identifies the auto-terms in the time-frequency plane, and can do this for mono- and multi-component signals with linear or curved time-frequency supports. TFCA is thus an effective, highresolution signal analysis technique that can yield the global distribution of uncontaminated components in the form of

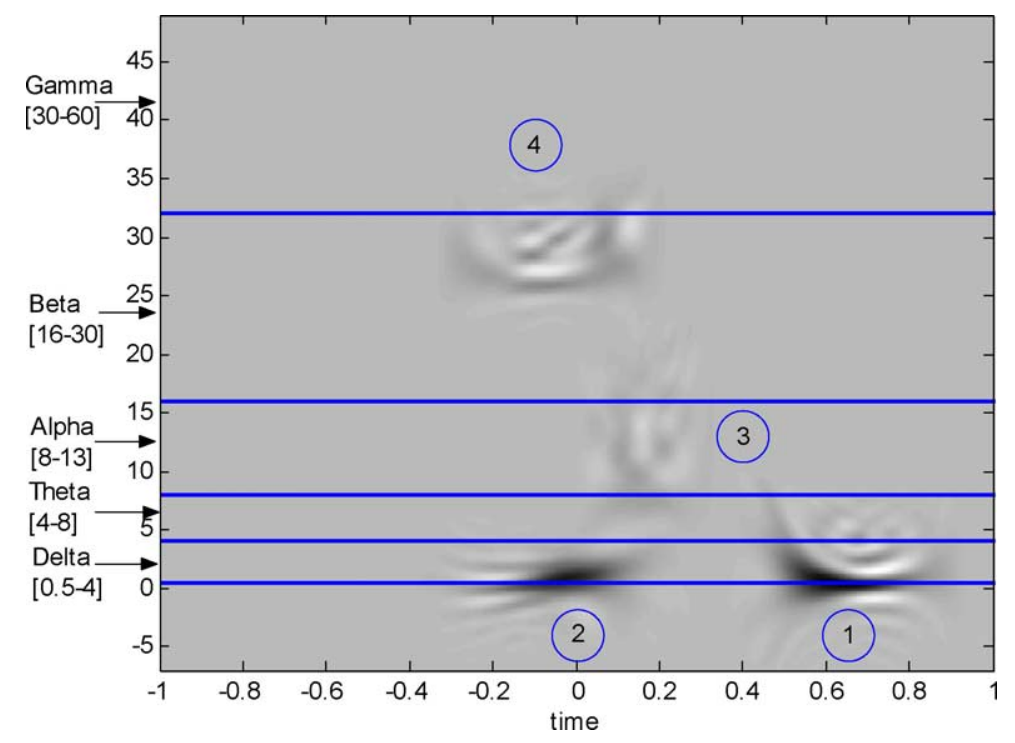

Fig. 11. Comparison of the conventional frequency limits of oscillatory components and the components obtained with TFCA for an ERP evoked by deviant stimuli in a trial subject. $\mathrm{X}$-axis: time in seconds; $\mathrm{Y}$-axis: frequency in $\mathrm{Hz}$. A linearly increasing frequency weighting function was used beyond $20 \mathrm{~Hz}$ in the superposition of the components in order to keep the weaker components visible beside the stronger ones. The numbers near to the components denote the order of the extraction. The locations of conventional frequency ranges are specified on the $Y$-axis. 
spatially and temporally integrated, time-varying oscillatory activity of various frequencies. TFCA seems therefore an appropriate tool for studying the intricate machinery of the human brain.

Recent work on brain neuroelectricity stresses the importance of single sweep analysis. Jansen et al. (2003) pointed out that ensemble averages will not resemble single trial responses. Likewise, single trial responses are not amplitude scaled versions of ensemble averages. Makeig (2002) showed that by means of an adequate analysis of single trials, dynamic consistencies between features of EEG averages (ERPs) and event-related changes in EEG signals can be found. The recently developed piecewise Prony method (Garossi and Jansen, 2000) has proven to be useful in decomposing nonstationary signals into a sum of oscillatory components with time-varying frequency, amplitude, and phase characteristics. The method could show the temporal profile of poststimulus signal changes in single-trial evoked potentials. A goal for the future studies should thus be to test the utility of TFCA on single sweep ERPs that have been obtained under different paradigms, and in different states of consciousness. Such studies might help to gain new insights into the oscillatory dynamics of the brain during different cognitive operations.

\section{References}

Adrian ED. Olfactory reactions in the brain of the hedgehog. J Physiol 1942:100:459-73.

Almeida LB. The fractional Fourier transform and time-frequency representations. IEEE Trans Signal Process 1994;42:3084-91.

Arıkan O, Özdemir AK, Tüfekçi DI, Çakmak ED, Karakaş S. A new technique for joint time-frequency analysis of event-related signals of the brain: time-frequency component analyzer (TFCA). Fifth Annual Conference of the EEG \& Clinical Neuroscience Society (ECNS). Clin EEG Electroencephalogr 2003;34(3):170.

Baraniuk RG, Jones DL. A signal-dependent time-frequency representation: optimal kernel design. IEEE Trans Signal Process 1993;41:1589-601.

Baraniuk R, Coates M, Steeghs P. Hybrid linear/quadratic time-frequency attributes. IEEE Trans Signal Process 2001;49:760-6.

Başar E. EEG-brain dynamics: relation between EEG and brain evokedpotentials. Amsterdam: Elsevier; 1980.

Başar E. Brain function and oscillations I: brain oscillations. Principles and approaches. Heidelberg: Springer-Verlag; 1998.

Başar E. Brain function and oscillations II: integrative brain function. Neurophysiology and cognitive processes. Heidelberg: SpringerVerlag; 1999.

Başar E, Başar-Eroğlu C, Karakaş S, Schürmann M. Brain oscillations in perception and memory. In: Chiarenza GA, editor. Proceedings of the Ninth World Congress of the International Organisation of Psychophysiology, vol. 35; 2000. p. 95-124.

Başar E, Demiralp T, Schürmann M, Başar-Eroğlu C, Ademoğlu A. Oscillatory brain dynamics, wavelet analysis and cognition. Brain Lang 1999;66(1):146-83.

Başar E, Özgören M, Başar-Eroğlu C, Karakaş S. Superbinding: spatio-temporal oscillatory dynamics. Theory Biosci 2003;121:37185.

Başar E, Ungan P. A component analysis and principles derived for the understanding of the evoked potentials of the brain: studies in the hippocampus. Kybernetik 1973;12:133-40.
Başar-Eroğlu C, Başar E, Demiralp T, Schürmann M. P300-response: possible psychophysiological correlates in delta and theta frequency channels. A review. Int J Psychophysiol 1992;13:161-79.

Berger H. Uber des elektroenkephalogram. Arch Psychiatry Nervenkr 1929;87:527-70.

Boashash B, O'Shea P. Use of the cross Wigner-Ville distribution for estimation of instantaneous frequency. IEEE Trans Signal Process 1993;41:1439-45.

Boudreaux-Bartels GF, Parks TW. Time-varying filtering and signal estimation using Wigner distribution synthesis techniques. IEEE Trans Acoust Speech Signal Process 1986(34):442-51.

Brandt ME, Jansen BH. The relationship between prestimulus alpha amplitude and visual evoked potential amplitude. Int J Neurosci 1991;61:261-8.

Brown MK, Rabiner LR. An adaptive, ordered, graph search technique for dynamic time warping for isolated word recognition. IEEE Trans Acoust Speech Signal Process 1982;30:535-44.

Coates M, Fitzgerald W. Time-frequency signal decomposition using energy mixture models. Proc IEEE Int Conf Acoust Speech Signal Process 2000;II:633-6.

Choi HI, Williams WJ. Improved time-frequency representation of multicomponent signals using exponential kernels. IEEE Trans Acoust Speech Signal Process 1989;37:862-71.

Cohen L. Time-frequency distributions: a review. Proc IEEE 1989; 77(7):941-81

Cohen L. Time-frequency analysis. Englewood Cliffs, NJ: Prentice-Hall; 1995.

Cook EW 3rd, Miller GA. Digital filtering: background and tutorial for psychophysiologists. Psychophysiology 1992;29:350-67.

Czerwinski RN, Jones DL. Adaptive cone-kernel time-frequency analysis. IEEE Trans Signal Process 1995;43:1715-9.

Dawson GD. A summation technique for the detection of small evoked potentials. Electroencephalogr Clin Neurophysiol 1954;6:65-84.

Demiralp T, Ademoğlu A, Istefanopulos Y, Başar-Eroğlu C, Başar E. Wavelet analysis of oddball P300. Int J Psychophysiol 2001;39:221-7.

Demiralp T, Ademoğlu A, Schürmann M, Başar E. Wavelet analysis of brain waves. In: Başar E, editor. Brain function and oscillations I: brain oscillations. Principles and approaches. Heidelberg: SpringerVerlag; 1998.

Demiralp T, Ademoğlu A, Schürmann M, Başar-Eroğlu C, Başar E. Detection of P300 in single trials by the wavelet transform (WT). Brain Lang 1999;66:108-28.

Donchin E, Coles MGH. Is the P300 component a manifestation of context updating? Behav Brain Sci 1988;11:357-74.

Donchin E, Karis D, Bashore TR, Coles MGH, Gratton G. Cognitive psychophysiology and human information processing. In: Coles MGH, Donchin E, Porges SW, editors. Psychophysiology: systems, processes and applications. New York: Guilford Press; 1986. p. 244-66.

Durak L, Arıkan O. Short-time Fourier transform: two fundamental properties and an optimal implementation. IEEE Trans Signal Process 2003;51(5):1231-42.

Erden MF, Kutay MA, Ozaktas HM. Repeated filtering in consecutive fractional Fourier domains and its application to signal restoration. IEEE Trans Signal Process 1999;47:1458-62.

Farwell LA, Martinerie JM, Bashore TR, Rapp PE, Goddard PH. Optimal digital filters for long-latency components of the event-related brain potential. Psychophysiology 1993;30:306-15.

Garossi V, Jansen BH. Development and evaluation of the piecewise Prony method for evoked potential analysis. IEEE Trans Biomed Eng 2000;47(12):1549-54.

Hlawatsch F, Flandrin P. The interference structure of the Wigner distribution and related time-frequency signal representations. In: The Wigner distribution - theory and applications in signal processing. Amsterdam: Elsevier; 1997 p. $59-133$.

Hlawatsch F, Matz G, Kirchauer H, Kozek W. Time-frequency formulation, design, and implementation of time-varying optimal filters for signal estimation. IEEE Trans Signal Process 2000;48:1417-32. 
Hlawatsch F, Kozek W. Time-frequency projection filters and timefrequency signal expansions. IEEE Trans Signal Process 1994; 42:3321-34.

Hlawatsch F, Costa AH, Krattenthaler W. Time-frequency signal synthesis with time-frequency extrapolation and don't-care regions. IEEE Trans Signal Process 1994;42:2513-20.

Humphreys DG, Kramer AF. Toward a psychophysiological assessment of dynamic changes in mental overload. Hum Factors 1994;36:3-22.

Jansen B, Agarwal A, Hegde A, Boutros NN. Phase synchronization of the ongoing EEG and auditory EP generation. Clin Neurophysiol 2003;114:79-85.

Jervis BW, Nichols MJ, Johnson TE, Allen E, Hudson NR. A fundamental investigation of the composition of auditory evoked potentials. IEEE Trans Biomed Eng 1983;30:43-9.

Johnson R. The amplitude of the P300 component of the event-related potentials: a review and synpaper. Advances in psychophysiology: a research annual, vol. 3. Greenwich: JAI Press; 1988.

Jones DL, Baraniuk RG. An adaptive optimal-kernel time-frequency representation. IEEE Trans Signal Process 1995;43:2361-71.

Kamarajan C, Porjesz B, Jones KA, Choi K, Chorlian DB, Padmanabhapillai A. The role of brain oscillations as functional correlates of cognitive systems: a study of frontal inhibitory control in alcoholism. Int J Psychophysiol 2004;51(2):155-80.

Karakaş S. A descriptive framework for information processing: an integrative approach. Brain alpha activity: new aspects and functional correlates. Int J Psychophysiol 1997;26:353-68.

Karakaş S, Başar E. Early gamma response is sensory in origin: a conclusion based on cross-comparison of results from multiple experimental paradigms. Int J Psychophysiol 1998;31(1):13-31.

Karakaş S, Erzengin OU, Başar E. A new strategy involving multiple cognitive paradigms demonstrates that ERP components are determined by the superposition of oscillatory responses. Clin Neurophysiol 2000a; 111:1719-32.

Karakaş S, Erzengin OU, Başar E. The genesis of human event-related responses explained through the theory of oscillatory neural assemblies. Neurosci Lett 2000b;285:45-8.

Katkovnik V, Stankovic L. Instantaneous frequency estimation using the Wigner distribution with varying data-driven window length. IEEE Trans Signal Process 1998;46:3215-25.

Kolev V, Yordanova J. Analysis of phase-locking is informative for studying event-related EEG activity. Biol Cybern 1997;96:22935.

Kramer AF, Strayer DL. Assessing the development of automatic processing: an application of dual-track and event-related brain potential methodologies. Biol Psychol 1988;26:231-67.

Kwok HK, Jones DL. Improved instantaneous frequency estimation using an adaptive short-time Fourier transform. IEEE Trans Signal Process 2000;48:2964-72.

Makeig S, Westerfield M, Jung TP, Enghoff S, Townsend J, Courchesne E, et al. Dynamic brain sources of visual evoked responses. Science 2002;295(5555):690-4.

Makeig S. Response: event-related brain dynamics-unifying brain electrophysiology. Trends Neurosci 2002;25(8):390.

McHale TJ, Boudreaux-Bartels GF. An algorithm for synthesizing signals from partial time-frequency models using the cross Wigner distribution. IEEE Trans Signal Process 1993;41(5):1986-90.

Meda N. Transversal filters with nonuniform tap spacing. IEEE Trans Circuits Syst 1980;27:1-11.

Mergenau H, Hill RN. Correlation between measurements in quantum theory. Prog Theor Phys 1961;26:722-38.

Mountcastle V. Preface. In: Induced rhythms of the brain. Berlin: Birkhauser; 1992.

Naatanen R. Processing negativity: an evoked potential reflection of selective attention. Psychol Bull 1982;92(3):605-40.

Naatanen R. The role of attention in auditory information processing as revealed by event-related potentials and other brain measures of cognitive function. Behav Brain Sci 1990;13(2):201-88.
Naatanen R. Attention and brain function. London: Lawrence Erlbaum Associates; 1992.

Naatanen R, Schröger E, Karakaş S, Tervaniemi M, Paavilainen P. Development of a memory trace for a complex sound in the human brain. Neuroreport 1993;4:503-6.

Ozaktas HM, Arıkan O, Kutay MA, Bozdagi G. Digital computation of the fractional Fourier transform. IEEE Trans Signal Process 1996;44:2141-50.

Özdemir AK. Time-frequency component analyzer. Unpublished doctoral dissertation, Bilkent University, Ankara, Turkey, 2003.

Özdemir AK, Arıkan O. Efficient computation of the ambiguity function and Wigner distribution on arbitrary line segments. IEEE Trans Signal Process 2001;49:381-93.

Özdemir AK, Arıkan O. A high-resolution time-frequency representation with significantly reduced cross-terms. Proc IEEE Int Conf Acoust Speech Signal Process 2000;2:693-6.

Özdemir AK, Durak L, Arıkan O. High-resolution time-frequency analysis based on fractional domain warping. Proc IEEE Int Conf Acoust Speech Signal Process 2001;6:3553-6.

Page CH. Instantaneous power spectra. J Appl Phys 1952;23:103-6.

Parvin C, Torres F, Johnson E. Synchronization of single evoked response components: estimation and interrelation of reproducibility measures. In: Rhythmic EEG activities and cortical functioning. Amsterdam: Elsevier; 1980.

Picton TW, editor. Human event-related potentials. Handbook of electroencephalography and clinical neurophysiology. Amsterdam: Elsevier; 1988.

Picton TW, Hillyard SA, Krausz HI, Galambos R. Human auditory evoked potentials. I. Evaluation of components. Electroencephalogr Clin Neurophysiol 1974;36:176-90.

Polich J, Kok A. Cognitive and biological determinants of P300: an integrative review. Biol Psychol 1995;41:103-46.

Polich J, Margala C. P300 and probability: comparison of oddball and single stimulus paradigms. Int J Psychophysiol 1997;25:16976.

Porjesz B, Almasy L, Edenberg HJ, Wang K, Chorlian DB, Foroud T. Linkage disequilibrium between the beta frequency of the human EEG and a GABAA receptor gene locus. Proc Natl Acad Sci USA 2002;99(6):3729-33.

Rangaswamy M, Porjesz B, Chorlian DB, Wang K, Jones KA, Bauer LO. Beta power in the EEG of alcoholics. Biol Psychiatry 2002;52(8):831-42.

Rangaswamy M, Porjesz B, Chorlian DB, Wang K, Jones KA, Kuperman S. Resting EEG in offspring of male alcoholics: beta frequencies. Int J Psychophysiol 2004;51(3):239-51.

Ritter W, Paavilainen P, Lavikainen J, Reinikainen K, Alho K, Sams $\mathrm{M}$, et al. Event-related potentials to repetition and change of auditory stimuli. Electroencephalogr Clin Neurophysiol 1992;83:30621.

Rosso OA, Blanco S, Yordanova J, Kolev V, Figliola A, Schürmann M, et al. Wavelet entropy: a new tool for analysis of short-duration brain electrical signals. J Neurosci Methods 2001;105:65-75.

Röschke J, Mann K, Riemann D, Frank C, Fell J. Sequential analysis of the brain's transfer properties during consecutive REM periods. Electroencephalogr Clin Neurophysiol 1995;96:390-7.

Samar V, Bobardikar A, Raghuveer MR, Swarz K. Wavelet analysis of neuro-electric waveforms: a conceptual tutorial. Brain Lang 1999;66:7-60.

Sannita WG. Stimulus-specific oscillatory responses of the brain: a time/frequency-related coding process. Clin Neurophysiol 2000;111(4):565-83.

Sayers BM, Beagley HA, Henshall WR. The mechanism of auditory evoked EEG responses. Nature 1974;247(15):481-3.

Solodovnikov VV. Introduction to the statistical dynamics of automatic control systems. New York: Dover; 1960.

Sutton S, Braren M, Zubin J, John ER. Evoked potential correlates of stimulus uncertainty. Science 1965;150:1187-8. 
Tağluk ME, Çakmak ED, Karakaş S. Analysis of time-varying energy of brain responses to an oddball paradigm using short-term smoothed Wigner-Ville distribution. Int $\mathbf{J}$ Neurosci, in press.

Tağluk ME, Çakmak ED, Karakaş S. High resolution energy distribution of brain electrical activity obtained under the oddball paradigm. BIOMUT May 2002;239:13-9.

Tervaniemi M, Saarinen J, Paavilainen P, Danilova N, Naatanen R. Evoked potential correlates of stimulus uncertainty. Biol Psychol 1994;38:157-67.

Vincent L, Soille P. Watersheds in digital spaces: an efficient algorithm based on immersion simulations. IEEE Trans Pattern Anal Mach Intell 1991;13(6):583-98.

Wickens C, Kramer A, Vanasse L, Dionchin E. The performance of concurrent task: a psychophysiological analysis of the reci- procity of information processing resources. Science 1983;221:1080 2.

Winkler I, Paavilainen P, Naatanen R. Can echoic memory store two traces simultaneously? A study of event-related brain potentials. Psychophysiology 1992;29(3):337-49.

Wulich D, Plotkin EI, Swamy MNS. Synpaper of discrete time-varying null filters for frequency-varying signals using the time-warping technique. IEEE Trans Circuits Syst 1990;37:977-90.

Yordanova J, Kolev V. A single sweep analysis of the theta frequency band during auditory oddball task. Psychophysiology 1998;35(1):116-26.

Yordonova J, Kolev V, Rosso OA, Schürmann M, Sokowitz OW, Özgören $\mathrm{M}$, et al. Wavelet entropy analysis of event-related potentials indicates modality of independent theta dominance. J Neurosci Methods 2002;117:99-109. 